\title{
Assessment on the Achievable Throughput of Multi-Band ITU-T G.652.D Fiber Transmission Systems
}

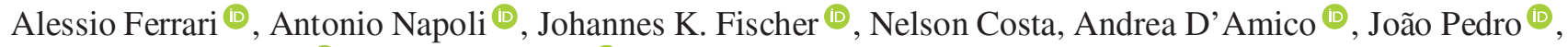 \\ Wladek Forysiak ${ }^{(\mathbb{1}}$, Erwan Pincemin ${ }^{\circledR}$, Andrew Lord, Alexandros Stavdas, Juan Pedro F.-P. Gimenez, \\ Gunther Roelkens ${ }^{(}$, Nicola Calabretta, Silvio Abrate ${ }^{(\mathbb{}}$, Bernd Sommerkorn-Krombholz, and Vittorio Curri
}

\begin{abstract}
Fiber-optic multi-band transmission (MBT) aims at exploiting the low-loss spectral windows of single-mode fibers (SMFs) for data transport, expanding by $\sim 11 \times$ the

available

bandwidth of C-band line systems and by $\sim 5 \times \mathrm{C}+\mathrm{L}$-band

line

systems'. MBT offers a high potential for cost-efficient throughput upgrades of optical networks, even in absence of available darkfibers, as it utilizes more efficiently the existing infrastructures. This represents the main advantage compared to approaches such as multi-mode/-core fibers or spatial division multiplexing. Furthermore, the industrial trend is clear: the first commercial $\mathbf{C}+\mathbf{L}$-band systems are entering the market and research has moved toward the neighboring $\mathrm{S}$-band. This article discusses the potential and challenges of MBT covering the ITU-T optical bands $\mathrm{O} \rightarrow$
\end{abstract}

L.

MBT performance is assessed by addressing the generalized SNR (GSNR) including both the linear and non-linear fiber propagation effects. Non-linear fiber propagation is taken into account by computing the generated non-linear interference by using the generalized Gaussian-noise (GGN) model, which takes into account the

Antonio Napoli and Bernd Sommerkorn-Krombholz are with the Infinera, 81541 Munich, Germany (e-mail: anapoli@infinera.com; bsommerkorn@infinera.com)

Johannes K. Fischer is with the Fraunhofer Institute for Telecommunications Heinrich Hertz Institute, 10587 Berlin, Germany (e-mail: johannes.fischer@hhi.fraunhofer.de).

Nelson Costa and João Pedro are with Infinera Portugal, 2790-078 Carnaxide, Portugal, and also with the Instituto de Telecomunicaçoes, IST, UL, Lisbon 1049-001, Portugal (e-mail: jpedro@ infinera.com).

Wladek Forysiak is with the Aston Institute of Photonic Technologies, Birmingham B4 7ET, U.K. (e-mail: w.forysiak@aston.ac.uk).

Erwan Pincemin is with Orange, 22300 Lannion, France (e-mail: erwan.pincemin@orange.com).

Andrew Lord is with the British Telecommunications, Ipswitch IP53RE, U.K. (e-mail: andrew.lord@bt.com).

Alexandros Stavdas is with the University of Peloponnese, 22100 Tripoli, Greece (e-mail: astavdas@ hotmail.com).

Juan Pedro F.-P. Gimenez is with Telefonica, 28050 Madrid, Spain (e-mail: juanpedro.fernandez-palaciosgimenez@telefonica.com).

Gunther Roelkens is with the Gent University, 9000 Gent, Belgium (e-mail: gunther.roelkens@ugent.be).

Nicola Calabretta is with TU/e 5612 AZ Eindhoven, The Netherlands (e-mail: n.calabretta@tue.nl).

Silvio Abrate is with Links Foundation, 10138 Torino, TO, Italy (e-mail: silvio.abrate@linksfoundation.com). interaction of non-linear fiber propagation with stimulated Raman scattering (SRS), and in general considers wavelength-dependent fiber parameters. For linear effects, we hypothesize typical components' figures and discussion on components' limitations, such as transceivers,' amplifiers' and filters' are not part of this work. We focus on assessing the transmission throughput that is realistic to achieve by using feasible multi-band components without specific optimizations and implementation discussion. So, results are meant to address the potential throughput scaling by turning-on excess fiber transmission bands. As transmission fiber, we focus exclusively on the ITU-T G.652.D, since it is the most widely deployed fiber type worldwide and the mostly suitable to multi-band transmission, thanks to its ultra-wide low-loss single-mode high-dispersion spectral region. Similar analyses could be carried out for other single-mode fiber types. We estimate a total single-fiber throughput of $450 \mathrm{~Tb} / \mathrm{s}$ over a distance of $50 \mathrm{~km}$ and $220 \mathrm{~Tb} / \mathrm{s}$ over regional distances of $600 \mathrm{~km}: \sim 10 \times$ and $8 \times$ more than C-band transmission respectively and $\sim 2.5 \times$ more than full $\mathrm{C}+\mathrm{L}$.

Index Terms-Multi-band fiber transmission, high-capacity systems, elastic optical networks.

\section{INTRODUCTION}

$\mathbf{L}$ EGACY optical networks operated on the C-band and deploying direct-detection $10 \mathrm{G}$ optical WDM transmission technologies, could achieve a maximum throughput of $\sim 1 \mathrm{~Tb} / \mathrm{s} /$ fiber. Coherent optical technologies have dramatically improved the transmission scenario, and commercial transponders can now convey over C-band line-systems up to $38.4 \mathrm{~Tb} / \mathrm{s} /$ fiber [1], by implementing a $38 \times$ transmission throughput increase, keeping the transmission infrastructure unchanged. On the other hand, it is predicted that IP traffic demand will continue to grow at a compound annual growth rate (CAGR) as large as 26\% [2] for back-bone networks, and it might be even larger for metro scenarios and data center interconnect (DCI) [2]. In addition, $5 \mathrm{G}$ and high-capacity access traffic as well as machine-to-machine communication, together with the expansion of cloud services will further load the backbone optical network infrastructure. Consequently, the per-fiber throughput enabled by the coherent transmission technologies is not sufficient anymore to support the envisioned IP traffic explosion, and alternative solutions must be found; with a firm request from Carriers to fully exploit the installed transmission equipment in order to maximize returns from investments [3]. 
Two different approaches have been proposed to upgrade infrastructures to cope with these requirements: spatial division multiplexing (SDM) with multi-fiber (MF) or multi-core/mode fiber (MMF/MCF) transmission; and multi-band transmission (MBT), which more efficiently exploits the available spectrum of a single fiber. MBT and SDM are not mutually exclusive, as MBT is a technique that maximizes the per-fiber transmission, that can be eventually combined with SDM by activating additional fibers, when needed. For both MF and MBT, transport platforms are commercially available, while MMF/MCF transmission needs complex multiple-input-multiple-output (MIMO) transceivers not yet commercially available. SDM can be implemented following two main strategies: I) by using the available dark-fibers, i.e., MF transmission [4] or II) by following the much costlier operation of rolling out new optical fiber infrastructures [5], consisting of MF/MMF/MCF. The MMF/MCF approach leads to a potential capacity in the Petabit/s/fiber range [6], [7], but it requires to establish a new transmission ecosystem with huge CAPEX efforts. Moreover, the required technologies and standardization lack of maturity for a near-term commercial deployment.

On the other hand, SDM with MF transmission is the most widely adopted solution in case of available dark-fibers as it relies on mature and cost-effective technologies by replicating C-band line systems. Nevertheless, in absence of available dark-fibers, techno-economics address the operators towards postponing such as solution that would require to install or lease new cables, with large CAPEX efforts and operational delays. If ducts are full, the cost for a new fiber roll-out is $\sim 25 \mathrm{k} € \backslash \mathrm{km}$

in

rural areas, and up to $\sim 500 \mathrm{k} € \backslash \mathrm{km}$ in metropolitan areas.

\section{SDM}

with MF transmission is highly costly also for network operators leasing fibers, that occurs when: I) they manage networks abroad $;{ }^{1}$ II) they lease infrastructures owned by incumbent operators; III) they cannot afford to deploy the new fibers. Moreover, engineering works may imply large and unpredictable delays because of needs for local authorities approvals, and negotiations, in general. In summary, MBT is an attractive solution to support the continuous growth in fiber transmission demand, as it postpones fiber roll-outs, and maximizes the return-on-investments on existing infrastructures [8], and is a seamless and complementary solution to SDM.

MBT aims at enabling transmission beyond the C-band by opening up the $2^{\text {nd }}$ and $3^{\text {rd }}$ low-loss windows of single-mode fibers (SMF) as depicted in Fig. 1 of [10] for ITU-T G.652.A/D fibers. For the mostly and widely deployed ITU-T G.652.D fiber [11], the $\leq 0.4 \mathrm{~dB} / \mathrm{km}$ overall spectral region

ranges

from $1260 \mathrm{~nm}$ to, at least, $1625 \mathrm{~nm}$ for a total bandwidth of $\sim 53.5 \mathrm{THz}$, i.e., $\sim 11 \times$ the C-band. ${ }^{2}$ The optical

bands

$\{\mathrm{O}, \mathrm{E}, \mathrm{S}, \mathrm{C}, \mathrm{L}\}$ depicted in Fig. 1 are defined as per ITU [9]. Commercial MBT are targeting the upgrade of existing C-band line systems, first, with commercially available C+L-band systems, by capitalizing on the re-use of Erbium amplifier

\begin{tabular}{|c|c|c|c|c|c|}
\hline Band & O & $\mathbf{E}$ & S & C & $\mathbf{L}$ \\
\hline $\begin{array}{l}\text { Wavelength } \\
(\mathrm{nm})[9]\end{array}$ & $1260-1360$ & $1360-1460$ & $1460-1530$ & $\begin{array}{c}1530 \\
- \\
1565\end{array}$ & $\begin{array}{c}1565 \\
- \\
1625\end{array}$ \\
\hline C-band & & & & $35 \mathrm{~nm}$ & \\
\hline C+L-band & & & & & $\mathrm{nm}$ \\
\hline \begin{tabular}{|l|} 
All bands \\
\end{tabular} & & $365 \mathrm{nr}$ & & & \\
\hline
\end{tabular}

Fig. 1. ITU-T band definition for single-mode fiber [9].

technology also within the L-band [12]. This operation adds $\sim 7 \mathrm{THz}$ to the $\sim 5 \mathrm{THz}$ of the C-band for a total bandwidth of $\sim 11.5 \mathrm{THz}$. In a second step, the remaining bands will be considered for data transport, starting from the S-band [13]. In principle, all types of single-mode fibers could be utilized for MBT, but the available bandwidth will depend on the actual fiber characteristics, mainly on the cut-off frequency defining the single-mode spectral region. Fibers with hydroxyl ions $(\mathrm{OH})$ causing an absorption peak in the E-band such as ITU-T G-652.A/B would see a significantly lower capacity compared to modern, but already widely deployed ITU-T G-652.D. On the other hand, pure silica core fiber (PSCF) which, besides having a zero $\mathrm{OH}$ absorption peak, presents a very large effective area - small non-linear coefficient - and very-low attenuation, will provide better performance than ITU-T G-652.D.

In $1990 \mathrm{~s}$, fiber manufacturer were able to purify silica from the OH ions, and the ITU-T G.652.D SMF fiber with no absorption peak was proposed, standardized in 2003 and commercialized to extend the transmission bandwidth of fiber systems [14], [15]. Consequently, the market share for ITU-T G-652.A/B have progressively decreased over the last 15 years. According to [16], the cumulative percentile of ITU-T G.652.D fiber - or compatible - deployed worldwide over the years 1993-2018 is $\sim 81 \%$ with an increase to up to $\sim 97 \%$ in $2013-2018$. Similar data are reported in [15]. Note that these figures refer average value worldwide. For example, in the USA, a large part of the nationwide backbone is made of non-zero dispersion-shifted fiber (ITU-T G.655) with large effective area, for which MBT is feasible only on $\mathrm{C}+\mathrm{L}$ bands. Among the fibers without the absorption peak, ITU-T G.652.D is the most deployed [11], [16] and is, therefore, the one we have considered in our analysis. Its measured physical parameters are reported in Fig. 2: the black line displays the dispersion whereas the green and blue lines show the attenuation for legacy ITU-T G.652.A (from the 80's) and ITU-T G.652.D optical fibers, respectively.

Fig. 2 shows that MBT has to cope with wavelength dependent channel characteristics: the dispersion coefficient $D(\lambda)$ ranges from $-5 \mathrm{ps} / \mathrm{nm} / \mathrm{km}$ to $\geq 20 \mathrm{ps} / \mathrm{nm} / \mathrm{km}$ (from O $\rightarrow$ L-band); whereas the fiber loss $\alpha(\lambda)$ ranges from $\sim 0.38 \mathrm{~dB} / \mathrm{km}$ (in O-band) to $\sim 0.18 \mathrm{~dB} / \mathrm{km}$ (in C-band). Consequently, to achieve a full optimization of transmission over such a wide spectrum, adaptation to the fiber characteristics on a per-channel and per-band basis is required. For instance, different modulation formats might be assigned to different transmission bands as proposed in [17]. Alternatively, probabilistic shaping and rateadaptive forward error correction (FEC) could be used to achieving a finer granularity [18].

\footnotetext{
${ }^{1}$ In this case, they are subjected to the host country regulations, which usually do not allow foreign companies to freely deploy new fibers.

${ }^{2}$ Note that commercial C-band systems occupy wider bandwidth than ITU
} 


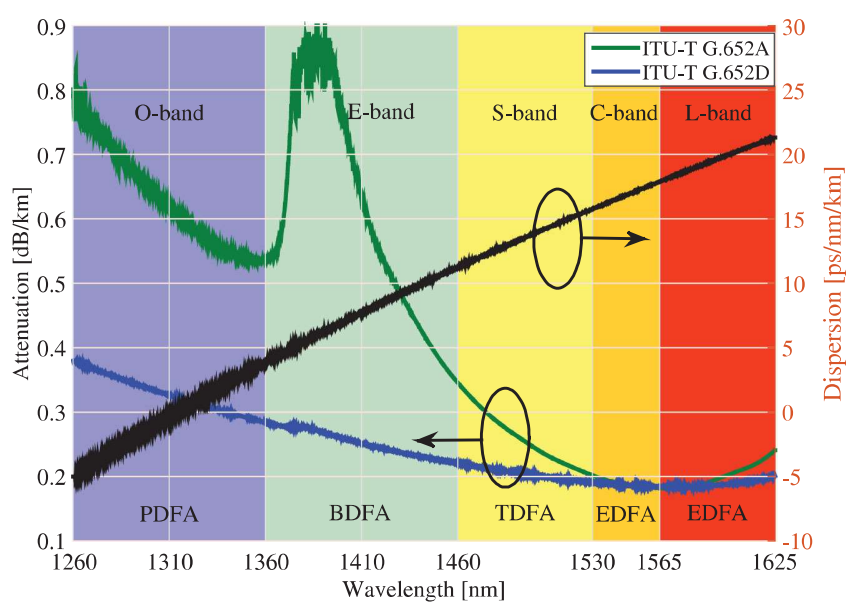

Fig. 2. Measured attenuation (green and blue) and dispersion coefficient (black) for ITU-T G.652.A and ITU-T G.652.D fibers. DFA stands for doped fiber amplifiers, and the considered doping materials are listed in Table II.

In the following paragraphs, we summarizes goals, assumptions and results of this article.

Goals: We aim at assessing the achievable throughput on a single mode fiber transmission system when relying on MBT on ITU-T G.652.D fiber. We assume the deployment of 32 Gbaud ideal rate-variable transceivers [18] on the $50 \mathrm{GHz}$ WDM fixed grid, so, results are not to meant as ultimate capacity assessment, but as reasonable figures achievable by turning-on additional bands in MBT, with the aim to assess the band-by-band throughput scaling-up factor referred to the C-band's. We focus the analysis on limitations introduced by fiber propagation, so we do to address specific components and control-software optimizations.

Assumptions: As performance meter, we use the generalized signal-to-noise ratio (GSNR) [19], [20] that includes both the accumulated ASE noise introduced by amplifiers to counteract fiber loss and SRS spectral tilt, and the non-linear interference (NLI) generated by fiber non-linear propagation by using the GNPy library [21] as it has been extensively validated [22]. For the NLI calculation we follow a completely disaggregated approach where each fiber span introduces its NLI contribution independently. For the NLI contribution calculation, we use the generalized Gaussian noise (GGN)-model [23]-[27] that together with the proven conservative accuracy of the GNmodel [20] considers the effects of spectral and spatial variations of fiber loss and its interaction with NLI generation [23]-[27]. As showed by results of this work, these effects, mainly induced by the SRS, are dominant when the transmission band approaches or exceeds the SRS peak, so when the transmission band occupancy approaches $10 \mathrm{THz}$. This article extends the work reported in [28], [29], precisely by including SRS effects while evaluating the evolution of signal power along the optical fiber and its interaction with fiber non-linearities - the generation of nonlinear interference (NLI). The goal of this work is to assess the MBT potentialities focusing on limitations introduced by fiber propagation, so, we deliberately suppose to rely on ideal amplifying and filtering components as well as ideal rate-variable (flexible) transceivers, by neglecting impairments arising from non ideal components. Thus, we suppose amplifiers delivering a flat per-band gain and noise-figure followed by ideal gain flattening filters. For per-band values of noise-figure we refer to feasible amplifying technologies as reported in literature. As power control strategy, we suppose the sub-optimal per-band flat launch power at every fiber span and the per-band power spectral density is computed out by applying the local optimization for a global optimization (LOGO) strategy to each band [30]. We are fully aware of the importance of analyzing the components' limitations and of the need to realize an ecosystem of devices enabling end-to-end transmission [31], e.g., optical MBT amplifiers and filters, as reported in Section II. But such analyses, as well as techno-economics, would be carried-out on top of fiber propagation potentialities in order to keep separated the potentialities of different design leverages. Some technologies are indicated merely with the objective of providing a strategy to implement a pay-as-you-grow approach for system upgrade.

Results: We derive, by estimating the GSNR, the total troughtput per band for the following scenarios: I) DCI-like, i.e., $\leq 80 \mathrm{~km}$; II) metro networks $\in\{150 \mathrm{~km}, 300 \mathrm{~km}\}$; and III) extended metro \& regional networks, i.e., up to $600 \mathrm{~km}$. We also assess the impact of using the O-band for Raman pumping instead of exploiting it for data transmission, to enhance the performance of the E-band, and potentially boosting the capacity of medium-long reach links.

The article is organized as follows. In Section II, we discuss the potentialities and challenges together with a possible road-map for the development of MBT systems. Section III describes the methodology used for the presented analyses. Next, Section III presents the considered system configurations and scenarios, and highlights the main assumptions for throughput evaluation. Afterwards, Section IV discusses on numerical analyses and presents results as maximum achievable data-rate per channel and throughput per band. Finally, Section V draws conclusions and addresses future investigations.

\section{Multi-Band Optical Systems: Potentialities, Challenges, AND RoAD-MaP}

Fig. 3 illustrates a generic MBT system: the multi-band transmitter and receiver (MB-TX, MB-RX) might employ a comb of tunable lasers, specific for each transmission band or, alternatively, over the entire $54 \mathrm{THz}$ low-loss spectrum. The multi-band MUX / DEMUX filters are capable of selecting any band, while the band amplifiers are supposed to be implemented by relying on ad-hoc doping glass materials, optimized for each band. For instance, we assume Praseodymium [32] for O-band; Bismuth [33] for E-band; Thulium [34] for S-band; and Erbium for C-and L-bands. A possible structure for multi-band re-configurable optical add/drop multiplexers (MB-ROADMs) and inline optical amplifiers is depicted in Fig. 3. System advantages may be obtained by using Raman amplification [35], also in MBT, by sacrificing some spectral portions from data transmission, as a large-enough guard band between the Raman pumps and channels must be guaranteed. Alternatively, we could completely remove data transmission from a band and use it to allocate the Raman pumps only. The latter is considered in ths 


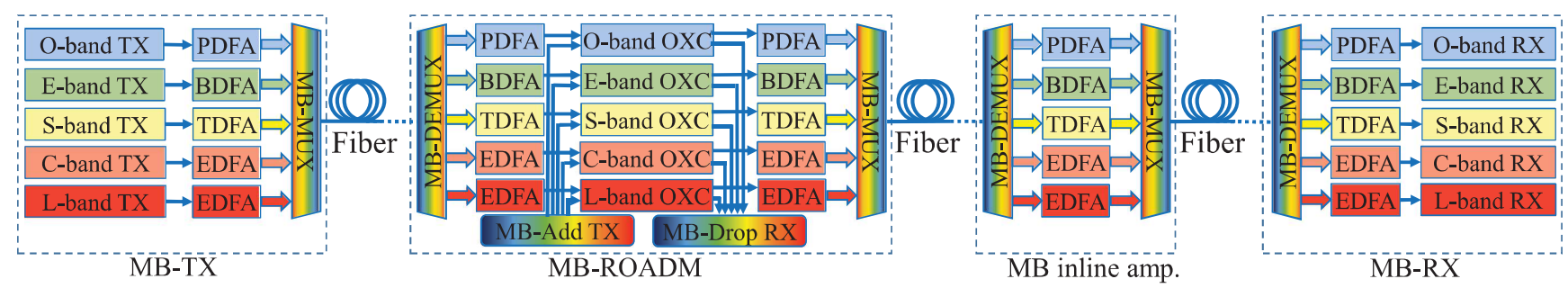

Fig. 3. Generic multi-band transmission system as considered within the numerical analysis. Transmitter (MB-TX); optical cross-connect (OXC); Receiver (MB-RX).

work, where all O-band specific components are removed from the link and replaced by counter-propagating Raman pumps. Finally, Raman amplifiers could be also realized as discrete components [36].

\section{A. Potentialities}

MBT is a realistic and practical approach to increase the capacity of optical networks in near future, because it efficiently uses the available optical fiber infrastructure, thus postponing new fiber roll-outs. Moreover, MBT enables a pay-as-you-grow approach also for the in-line network elements, e.g., filters, amplifiers. For example, some operators are in the process of upgrading their C-band systems with the L-band [37]. Although a techno-economic analyses is out-of-the-scope, we based our work on data received from the operators, which indicate MBT has high potential for capacity increase. In order to deliver a simple quantitative projection, we may assume $\{20,20,50\} \mathrm{Tb} / \mathrm{s}$ throughput for C-, L-band, all remaining bands, respectively; and $30 \%$ of traffic growth. From this, one can find out that after hypothetical 20 years growth at $30 \%,\{96,48,22\}$ fibers would be necessary in case of relying on $\mathrm{C}$-only, $\mathrm{C}+\mathrm{L}$ and all bands line systems, respectively. Therefore, it is clear that multiple fiber transmission is inevitable, but MBT is an effective technology to better exploit the infrastructure by enhancing the spatial efficiency in bit $/ \mathrm{s} / \mathrm{m}^{2}$ in ducts.

MBT opens up to novel leverages for traffic and network management. The broad spectrum enables to transmitting hundreds of channels with quite different optical performance, and this allows the existence of different classes of traffic among them. Another advantage of MBT is that it allows operators to manage less fibers with respect to SDM. For instance, while the O-band could carry short-reach traffic, e.g., $\leq 80 \mathrm{~km}$, long-haul (LH) traffic could be transported on the better performing $\mathrm{C}$ - or L-bands. In this context, a first assessment on the MBT potentialities, performing an iterative power optimization scheme for C-, S- and L-band, has been proposed in [38], [39]. In [38], a $150 \mathrm{~Tb} / \mathrm{s}$ capacity has been shown after $40 \mathrm{~km}$ by using S-, Cand L-bands. In this work, we show that for similar distances -
$50 \mathrm{~km}$, specifically - the throughput might triple by occupying the spectrum from O- to L-band.

\section{B. Challenges}

The main challenge for MBT is the low maturity of the key components. While $\mathrm{C}$-band devices have achieved high maturity, MB optical components are still at an early stage ${ }^{3}$. For example, first prototypes of MB-TX/MB-RX have been presented in [40], and multi-band amplifiers enabled new transmission records with fiber capacity of $115 \mathrm{~Tb} / \mathrm{s}$ in S-, C- and L-band as shown in [13] or achieving high performance in the case of hybrid EDFA+Raman amplifier for C+L [41]. MBT opens up an enormous spectrum for transmission, enabling the co-propagation of a large number of channels. This is a critical aspect for the network operators due to the considerably different transmission characteristics of each band, as shown in Fig. 2. Efficiently managing such a wide spectrum can be quite cumbersome, due to the high dependence of optical performance on the selected wavelength. An effective exploitation of the available capacity could be achieved by: I) deploying bandwidth variable transponders which can adapt their symbol rate/modulation format over the entire spectrum; and II) using an advanced control plane embedding an accurate performance model and a routing, modulation format and spectrum assignment algorithm that efficiently exploits the performance variations across the entire $54 \mathrm{THz}$ spectrum.

Table I list the potentialities and challenges of MBT in terms of pros and cons when compared to transmission solutions such as $\mathrm{MF}, \mathrm{MMF}$, and MCF.

\section{Road-Map Towards Multi-Band Systems}

Fig. 4 displays a possible road-map towards the adoption of MBT systems by network operators and service providers. Based on the currently available technology and, in particular, given the

\footnotetext{
${ }^{3}$ E.g., silicon photonics integrated circuits show high potential to realize costefficient MB-RX: a first discussion on the technology options and state-of-the-art of each multi-band component can be found in [31].
}

$$
\begin{aligned}
G_{\mathrm{NLI}}\left(L_{s}, f\right)= & \frac{16}{27} \gamma^{2} \iint_{-\infty}^{+\infty} G_{\mathrm{TX}}\left(f_{1}\right) G_{\mathrm{TX}}\left(f_{2}\right) G_{\mathrm{TX}}\left(f_{1}+f_{2}-f\right) \\
& \left|\int_{0}^{L_{s}} \exp \left(+j 4 \pi^{2}\left(f_{1}-f\right)\left(f_{2}-f\right)\left[\beta_{2}+\pi \beta_{3}\left(f_{1}+f_{2}\right)\right] \zeta\right) \frac{\rho\left(\zeta, f_{1}\right) \rho\left(\zeta, f_{1}+f_{2}-f\right) \rho\left(\zeta, f_{2}\right)}{\rho(\zeta, f)} \mathrm{d} \zeta\right|^{2} \mathrm{~d} f_{1} \mathrm{~d} f_{2}
\end{aligned}
$$


TABLE I

COMPARISON OF STRATEGIES FOR NETWORK CAPACITY EXPANSION

\begin{tabular}{|c|c|c|c|c|}
\hline Strategy & Main pros & Main cons & Maturity & Capacity \\
\hline Multi-fiber & $\begin{array}{l}\text { 1) Mature technology } \\
\text { 2) Exploitation of available dark-fibers }\end{array}$ & $\begin{array}{l}\text { 1) Costly new fiber deployment } \\
\text { 2) Complex management of SDM net- } \\
\text { works }\end{array}$ & High & $\begin{array}{l}\text { Proportional to the } \\
\text { number of available } \\
\text { fibers }\end{array}$ \\
\hline $\begin{array}{l}\text { Multi-core / - } \\
\text { mode fiber }\end{array}$ & High capacity per optical fiber & $\begin{array}{l}\text { 1) New deployment of optical fibers } \\
\text { 2) Development of new components } \\
\text { 3) Complex management due to large } \\
\text { amount of WDM channels and dif- } \\
\text { ferent cores }\end{array}$ & $\begin{array}{l}\text { Significantly } \\
\text { low }\end{array}$ & $\begin{array}{l}\text { Proportional to the } \\
\text { number of available } \\
\text { fibers, number of cores } \\
\text { and modes }\end{array}$ \\
\hline $\begin{array}{l}\text { Multi-band } \\
\text { transmission }\end{array}$ & $\begin{array}{l}\text { 1) Exploits the full low-loss region of } \\
\text { SMF } \\
\text { 2) Maximizes previous investments }\end{array}$ & $\begin{array}{l}\text { 1) Development of new components for } \\
\text { O, E, and S-bands. } \\
\text { 2) Complex management due to large } \\
\text { amount of WDM channels }\end{array}$ & Low & $\begin{array}{l}\text { Up to } \sim 10 \times \text { the current } \\
\text { capacity per fiber }\end{array}$ \\
\hline
\end{tabular}

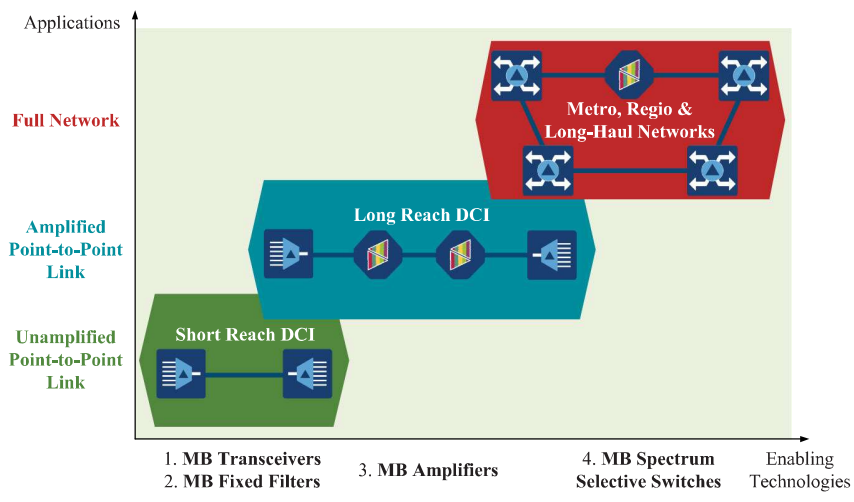

Fig. 4. Road-map towards the implementation of MBT systems.

initial absence of mature MB amplifiers, we foresee that the first application will involve mainly short-reach links. The maximum achievable distance of these systems will strictly depend on the channel power budget. At this stage, MB transceivers and fixedfilters will be the only pre-requisite. This first use case is very relevant because it covers a fast growing market sector, both in terms of demand and DCI applications. ${ }^{4}$ Once the technologies for MB amplifiers will be sufficiently mature, longer reach links, such as long-distance DCI and metro-aggregation networks, e.g., up to $300 \mathrm{~km}$, may become a reality. This will require additional CAPEX but, as MBT enables a pay-as-you-grow approach, the increased cost can be diluted over time. At this stage, it will be highly desirable that the amplification sites of the MB amplifiers coincide with the existing ones. At the final stage, regional and LH multi-band networks may become viable, enabled by mature MB amplifiers and ROADMs.

\section{Multi-BAND TRANSMission ANALYSIS}

In line systems using optical coherent technologies, the most limiting transmission impairments are the joint effect of the accumulation of ASE noise - introduced by the amplifiers and NLI disturbance generated within fiber propagation. Other effects, such as accumulated dispersion or polarization mode

\footnotetext{
${ }^{4}$ Solutions such as WDM 400ZR are now available on the market because of the high demand. It is worth to mention that the largest number of DCI links is well below $80 \mathrm{~km} \mathrm{[42].}$
}

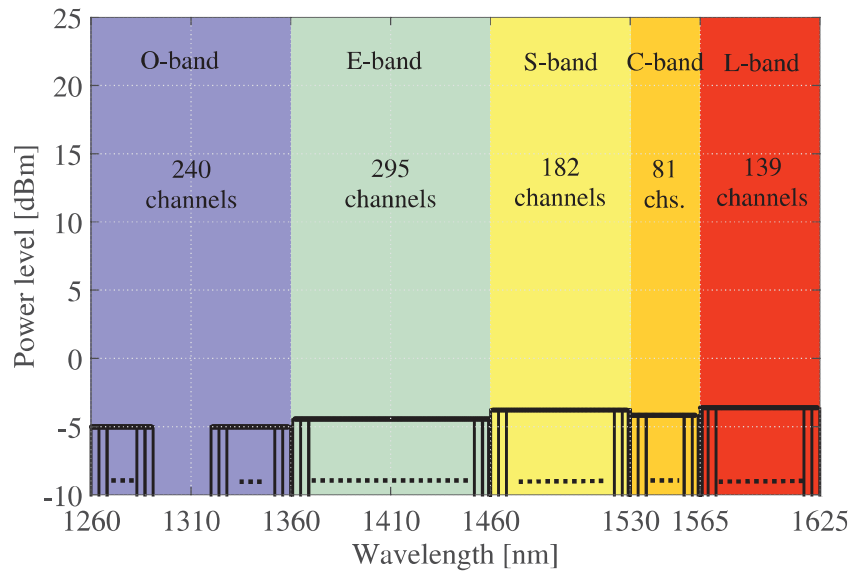

(a)

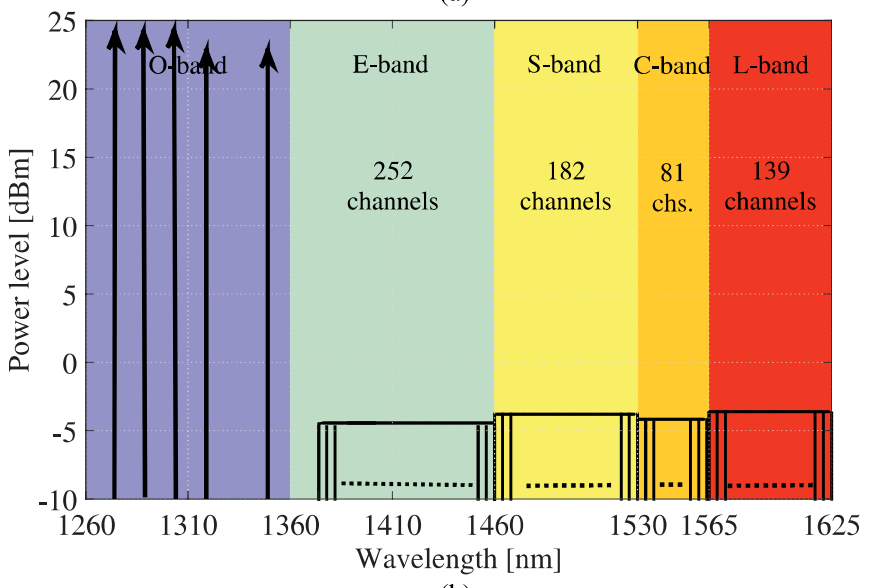

(b)

Fig. 5. Considered system configurations: a) Full MBT; b) O-band off, Raman in O-band, E $\rightarrow$ L-band.

dispersion (PMD) are mostly compensated by the DSP within the receiver. It is well accepted that the quality-of-transmission (QoT) figure of merit for the deployed lightpaths is given by the GSNR [19], [20] that includes both the effects of the accumulated ASE noise and NLI disturbance, defined as

$$
\mathrm{GSNR}=\frac{\mathrm{P}_{\mathrm{RX}}}{\mathrm{P}_{\mathrm{ASE}}+\mathrm{P}_{\mathrm{NLI}}}=\left(\mathrm{OSNR}^{-1}+\mathrm{SNR}_{\mathrm{NL}}^{-1}\right)^{-1}
$$


TABLE II

Considered PARAmeters Per BAND

\begin{tabular}{c|ccccc}
\hline Parameters / Band & 0 & $\mathrm{E}$ & $\mathrm{S}$ & $\mathrm{C}$ & $\mathrm{L}$ \\
\hline Wavelength range [nm] & $1260-1360$ & $1360-1460$ & $1460-1530$ & $1530-1565$ & $1565-1625$ \\
Frequency range [THz] & $220.59-238.10$ & $205.48-220.59$ & $196.08-205.48$ & $191.69-196.08$ & $184.62-191.69$ \\
Bandwidth [THz] & 17.25 & 14.81 & 9.13 & 4.13 & 6.96 \\
Number of channels & 240 & 296 & 182 & 82 & 139 \\
Central frequency [THz] & 229.07 & 212.79 & 200.65 & 193.89 & 188.07 \\
Type of amplifier & PDFA [32] & BDFA [33] & TDFA [34] & EDFA & EDFA \\
Amplifier's noise figure [dB] & 7 & 6 & 7 & 5.5 & 6 \\
Non-linear coefficient $\gamma[1 / \mathrm{W} / \mathrm{km}]$ & 1.6 & 1.5 & 1.4 & 1.3 & 1.28 \\
\hline
\end{tabular}

TABLE III

Transmitted Power Per Channel Per Band

\begin{tabular}{c|c|ccccc}
\hline & & $\mathrm{O}$ & $\mathrm{E}$ & $\mathrm{S}$ & $\mathrm{C}$ & $\mathrm{L}$ \\
\hline \multirow{2}{*}{ Full MBT } & $L_{S}=50 \mathrm{~km}$ & $-4.0 \mathrm{dBm}$ & $-3.4 \mathrm{dBm}$ & $-2.7 \mathrm{dBm}$ & $-3.1 \mathrm{dBm}$ & $-2.5 \mathrm{dBm}$ \\
& $L_{S}=75 \mathrm{~km}$ & $-0.9 \mathrm{dBm}$ & $-1.1 \mathrm{dBm}$ & $-1.0 \mathrm{dBm}$ & $-1.7 \mathrm{dBm}$ & $-1.0 \mathrm{dBm}$ \\
\hline \multirow{2}{*}{ O-band off and no Raman amplification } & $L_{S}=50 \mathrm{~km}$ & - & $-3.4 \mathrm{dBm}$ & $-2.7 \mathrm{dBm}$ & $-3.1 \mathrm{dBm}$ & $-2.5 \mathrm{dBm}$ \\
& $L_{S}=75 \mathrm{~km}$ & - & $-1.1 \mathrm{dBm}$ & $-1.0 \mathrm{dBm}$ & $-1.7 \mathrm{dBm}$ & $-1.0 \mathrm{dBm}$ \\
\hline \multirow{2}{*}{ Raman amplification in the O-band } & $L_{S}=50 \mathrm{~km}$ & - & $-4.2 \mathrm{dBm}$ & $-2.7 \mathrm{dBm}$ & $-3.1 \mathrm{dBm}$ & $-2.5 \mathrm{dBm}$ \\
& $L_{S}=75 \mathrm{~km}$ & - & $-1.9 \mathrm{dBm}$ & $-1.0 \mathrm{dBm}$ & $-1.7 \mathrm{dBm}$ & $-1.0 \mathrm{dBm}$ \\
\hline
\end{tabular}

where OSNR $=\mathrm{P}_{\mathrm{RX}} / \mathrm{P}_{\mathrm{ASE}}$ is the optical SNR measurable on the optical spectrum analyzer, $\mathrm{SNR}_{\mathrm{NL}}=\mathrm{P}_{\mathrm{RX}} / \mathrm{P}_{\mathrm{NLI}}$ is the non-linear SNR consider the effect of NLI only, $\mathrm{P}_{\mathrm{RX}}$ is the power of the channel under test (CUT), $\mathrm{P}_{\mathrm{ASE}}$ is the power of the ASE noise and $\mathrm{P}_{\mathrm{NLI}}$ is the power of the NLI. The GSNR corresponds to the error vector magnitude (EVM) [43] readable on the DSP-recovered constellation in case of absence of substantial phase-noise. Given the back-to-back transceiver characterization, the GSNR well predicts the BER, as extensively shown also in multi-vendor experiments using commercial products [20]. We suppose the controller to set the line operating at the LOGO [30], so the GSNR will be dominated by the ASE noise being OSNR $=1 / 2 \mathrm{SNR}_{\mathrm{NL}}$. The OSNR calculation is analytic based on the knowledge of gain and noise figures of amplifiers, together with power evolution per-frequency in fibers. While, for the NLI calculation, we rely on the GGN-model that generalized the GN-model in case of presence of substantial space and frequency variation of fiber loss - and gain, in case of Raman amplification.

In [44], the GN-model was introduced by applying to optical transmission using coherent technologies the concept already presented in the original paper [45]. The GN-model has subsequently been extensively validated, confirming that it can be employed for fast but still accurate network performance optimization [46]. Moreover, it was shown in [20] that it can be used fro a quick yet accurate and conservative QoT estimation in transmission over optical bandwidths as large as $3 \mathrm{THz}$. In case of a wider spectrum, the SRS becomes the most limiting effect as it induces a spectral tilt intra- and inter-band towards lower frequencies that can be avoided only with a guard-band as large as $15 \mathrm{THz}$, which is approximately the SRS bandwidth. Indeed, it has been experimentally shown [47] that the SRS-induced spectral tilt is the main effect in a $\mathrm{S}+\mathrm{L}$ system.

As the bandwidth for the inter-channel cross-talk including the modulation effect is quite limited, the SRS-induced amplitude disturbance is not a considerable impairment, as shown experimentally in [48]. In [49], an extension of the GN-model including the Raman amplification and assuming a flat spectral Raman effect has been proposed, and in [50] design strategies for optimal hybrid Raman/EDFA amplification have been derived [51]. However, such assumptions are not sufficiently general for MBT systems because, besides the variation of gain/loss with the space, also the variations in frequency are considerable. Thus, the GN-model has been generalized to the GGN-model [24], [26], [27] to accurately account for the frequency and space variations of gain/loss along the fiber spans and across the transmission bands as well as the wavelength dependence of dispersion, so, fully considering the SRS effects - crosstalk and amplification. In [52], [53], the GGN-model has been experimentally validated and, in [54], it has been proposed to estimate the QoT for point-to-point and network performances, considering optical bandwidths as large as $10 \mathrm{THz}$. Although GN-like models are usually quite accurate, especially in the C-band, their accuracy decreases in the presence of very-low fiber chromatic dispersion parameters. Nevertheless, in the case of ITU-T G.652 fibers, the close-to-zero dispersion parameter occurs at high frequencies, where the power depletion due to SRS is large, making NLI negligible with respect to ASE noise. The accuracy for GN-like models is also reduced when used for short-reach links, such as single-span systems. However, the estimation is conservative in this case. Thus, it can still be used to depict a general trend on the system capacity.

Eq. (1) shows the GGN-model formula estimating the NLI power spectral density $G_{\mathrm{NLI}}\left(L_{s}, f\right)$ generated by a wavelength division multiplexed (WDM) comb with a power spectral density $G_{\mathrm{TX}}(f)$ after transmission along a fiber span with length $L_{s}$, non-linear coefficient $\gamma$, dispersion parameter $\beta_{2}$ and dispersion slope $\beta_{3}$. The term $\rho(z, f)$ is the overall frequency and space dependent fiber loss profile. This parameter includes the non-flat fiber attenuation and the SRS: both Raman cross-talk and Raman amplification, if any. Details on the evaluation of $\rho(z, f)$ are reported in appendix A. In presence of Raman pumps, the SNR 


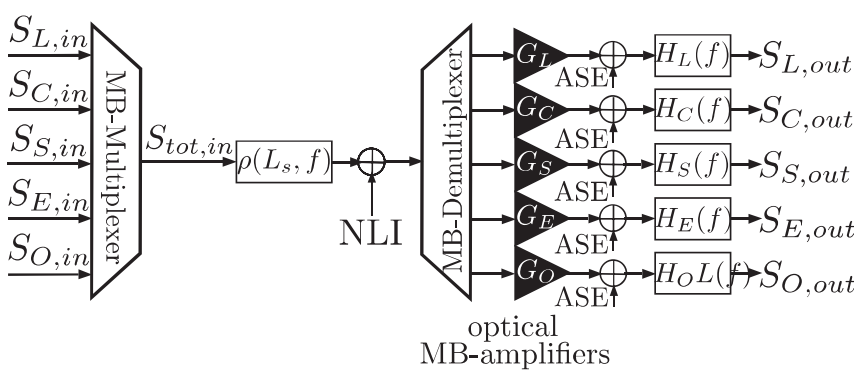

Fig. 6. Equivalent block diagram of a span.

TABLE IV

O-BAND RAMAN PUMP CONFIGURATION

\begin{tabular}{c|ccccc}
\hline Frequency $[\mathrm{THz}]$ & 222.22 & 227.27 & 229.89 & 232.56 & 235.29 \\
\hline Power $[\mathrm{mW}]$ & 200 & 200 & 350 & 350 & 350 \\
\hline
\end{tabular}

penalty introduced by the Rayleigh back-scattered pump-pump four-wave mixing (FWM) has been assessed according to [55]. Considering a Rayleigh backscattering coefficient $(\kappa)$ of $10^{-7} \frac{1}{m}$ and conservatively supposing large linewidth and possible variations in the pumps' wavelegths, a $2 \mathrm{THz}$ extra guard-band has been adopted to keep the pump-pump FWM always negligible. So, in presence of Raman pumps, the number of channels in the E-band is reduced from 295 to 252 . From Eq. (1), it is possible to calculate the final GSNR, at the optical fiber output. Further details on the GSNR computation are reported in Appendix B.

\section{A. The System Under Analysis}

A fully loaded WDM comb on MBT, ranging from the Oto the L-band is investigated, for a total occupied bandwidth of $\sim 53 \mathrm{THz}$. A $2 \mathrm{~nm}$ guard-band is assumed between adjacent bands for filtering purposes. ${ }^{5} \mathrm{~A}$ non-transmission bandwidth of $30 \mathrm{~nm}(5 \mathrm{THz})$ centered at the SMF zero-dispersion wavelength $(D(\lambda) \approx 0)$ is set and computed to maximize the O-band capacity as described in [28]. Table II reports the main parameters per band, showing that a total of $\sim 900$ channels can be transmitted in a MBT system when employing a $50 \mathrm{GHz}$ WDM grid. This amount of WDM channels is $\sim 10$ as large as the one of commercial systems, nevertheless the maximum resulting total power we are considering is $\leq 1.5 \mathrm{~W}$, for case with transmission over $\mathrm{E} \rightarrow \mathrm{L}$ with Raman pumps. This power level is well below, for example, the injected power in [56] or of the specification of commercial amplifiers [57]. On the other hand, it is important to mention that MBT requires major upgrades in the way optical systems will be certificated and standardized. In fact, current safety standards, e.g., IEC 60825-2 consider a maximum optical power up to $500 \mathrm{~mW}$ and telecommunication operators are obliged to respect this normative with their deployed system using Raman cards. To conclude this topic, it is important to mention that new standards will be required and new safety regulations and training for the workers installing the systems, will be needed before a MBT can be deployed. Table III reports the transmitted power per channel per band for each considered

\footnotetext{
${ }^{5}$ This is a benchmark value that is not yet feasible with mature filtering technologies
}

system configuration and for each span length, at the input of the fiber span. The power per channel within each band has been assumed as spectrally flat set as described in Section III-B. In this work, root raised-cosine shaped signals with a roll-off factor of 0.15 and a symbol-rate of 32 GBaud are assumed. The modulation format is adaptable - i.e., a bit-loading technique is supposed - and is determined by the GSNR value ${ }^{6}$ The line system is considered periodic and composed of $N_{s}$ spans of equal length $L_{s}$ and made of ITU-T G.652.D fiber whose fiber loss coefficient and the dispersion curve vs. wavelength are displayed in Fig. 2. The non-linear coefficient $\gamma$ is practically flat within each band, and the values are reported in Table II. Fig. 6 depicts the optical system under analysis. We assume to use a comb of transmitters specific for each transmission band. Then, a MB-MUX combines all channels in a unique WDM MBT comb and the resulting WDM signal is transmitted through the optical link. Every MB-DEMUX introduces $3 \mathrm{~dB}$ loss. A MB-DEMUX is used at the end of each fiber span to separate the different bands which are then amplified by different lumped optical amplifiers. Since the aim of the study is to provide a benchmark, ideally flat amplification, capable to sustaining the required power gain, is assumed. A gain flattening filter (GFF) is used after each band amplifier to restore the launched power profile at the input of the next span. At the end of the optical line, the WDM MBT comb is DEMUX'ed, amplified and, finally, detected. Amplifiers and filters are kept as simple as possible, because the aim of the work is to investigate whether the MBT is feasible given the assumed noise figure values of the amplifiers as present in the literature. For the target scenarios, from DCI to regional networks, we considered two values for the span length $L_{s}: 50 \mathrm{~km}$ and $75 \mathrm{~km}$.

\section{B. The Optical Transmitted Power}

We use a LOGO strategy [30], which assumes flat per-band power per channel, with the power level of each band set accordingly. Each band is evaluated independently of the others with the nominal values of the transmission band, i.e., the ones at the central frequency of each band, used as inputs for the power computation. We assume each lumped amplifier has a constant gain equal to the highest loss within its corresponding amplification band. In such a way, the lumped amplifiers plus GFFs act as MBT optical equalizers. The power spectral density at the input of each fiber span, when signals are transmitted from O- to L-band, is illustrated in Fig. 5(a).

\section{Throughput Maximization: A Trade-Off Between Available Bands and Physical Effects}

1) Ideal Flexible Transceiver: We assume ideal flexible transceivers, capable of completely exploiting the available GSNR, thus enabling the maximum feasible bit-rate, as shown in Section III-B of [58]. A total of $12 \%$ rate overhead is assumed, and this value takes into account for the FEC overhead and DSP pilots.

\footnotetext{
${ }^{6}$ Note that this is only one of the possible pair of modulation formats and grid. Clearly, in case of other modulation formats and symbol rates, the throughput will scale accordingly, but the trend will remain unchanged.
} 


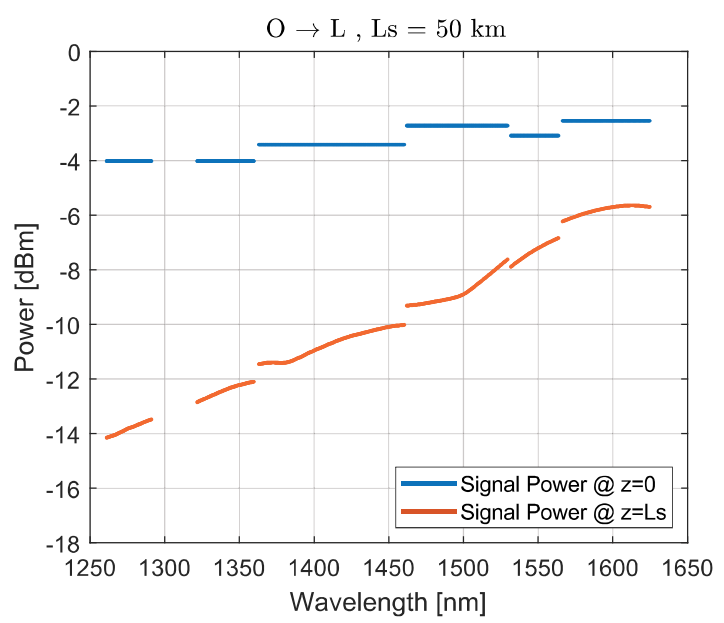

(a)

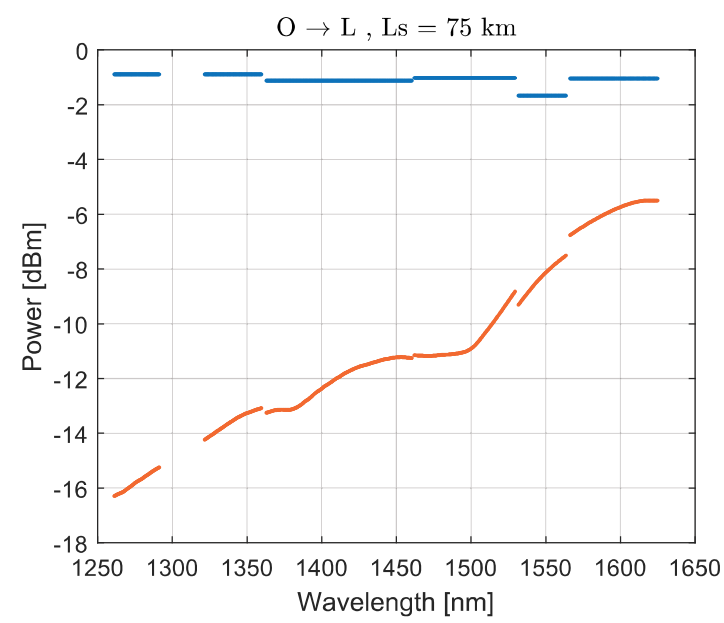

(b)

Fig. 7. Signal power at the beginning of the fiber $(z=0)$ and at the end $\left(z=L_{S}\right)$, for $L_{S}=50 \mathrm{~km}(\mathrm{a})$ and $L_{S}=75 \mathrm{~km}(\mathrm{~b})$.

TABLE V

AVERAGE SPECTRAL EFFiCIENCY PER BAND [BPS/Hz]

\begin{tabular}{|c|c|c|c|c|c|c|c|c|c|c|c|c|c|c|}
\hline & & \multicolumn{5}{|c|}{$\mathrm{O} \rightarrow \mathrm{L}$} & \multicolumn{4}{|c|}{ No Raman, $\mathrm{E} \rightarrow \mathrm{L}$} & \multicolumn{4}{|c|}{ Raman in $\mathrm{O}, \mathrm{E} \rightarrow \mathrm{L}$} \\
\hline & & $\mathrm{O}$ & $\mathrm{E}$ & S & $\mathrm{C}$ & $\mathrm{L}$ & $\mathrm{E}$ & S & $\mathrm{C}$ & $\mathrm{L}$ & E & S & $\mathrm{C}$ & $\mathrm{L}$ \\
\hline \multirow{4}{*}{$L_{s}=50 \mathrm{~km}$} & DCI & 8.7 & 10.0 & 10.0 & 9.9 & 9.4 & 10.0 & 10.3 & 9.9 & 9.6 & 11.2 & 11.1 & 10.8 & 10.6 \\
\hline & Metro & 6.9 & 8.2 & 8.3 & 8.1 & 7.7 & 8.2 & 8.5 & 8.1 & 7.8 & 9.4 & 9.3 & 9.0 & 8.8 \\
\hline & Extended Metro & 5.8 & 7.1 & 7.1 & 7.0 & 6.6 & 7.1 & 7.4 & 7.0 & 6.7 & 8.3 & 8.2 & 7.9 & 7.7 \\
\hline & Regional Network & 4.7 & 6.0 & 6.0 & 5.9 & 5.5 & 6.0 & 6.3 & 5.9 & 5.6 & 7.2 & 7.1 & 6.8 & 6.6 \\
\hline \multirow{4}{*}{$L_{s}=75 \mathrm{~km}$} & DCI & 6.8 & 8.5 & 8.7 & 8.5 & 7.8 & 8.5 & 9.0 & 8.5 & 7.9 & 9.3 & 9.3 & 8.9 & 8.7 \\
\hline & Metro & 5.7 & 7.4 & 7.6 & 7.4 & 6.7 & 7.4 & 7.9 & 7.4 & 6.8 & 8.2 & 8.1 & 7.8 & 7.6 \\
\hline & Extended Metro & 4.6 & 6.3 & 6.5 & 6.3 & 5.6 & 6.3 & 6.7 & 6.3 & 5.7 & 7.1 & 7.0 & 6.7 & 6.5 \\
\hline & Regional Network & 3.6 & 5.2 & 5.4 & 5.2 & 4.5 & 5.2 & 5.6 & 5.2 & 4.6 & 6.0 & 5.9 & 5.6 & 5.4 \\
\hline
\end{tabular}

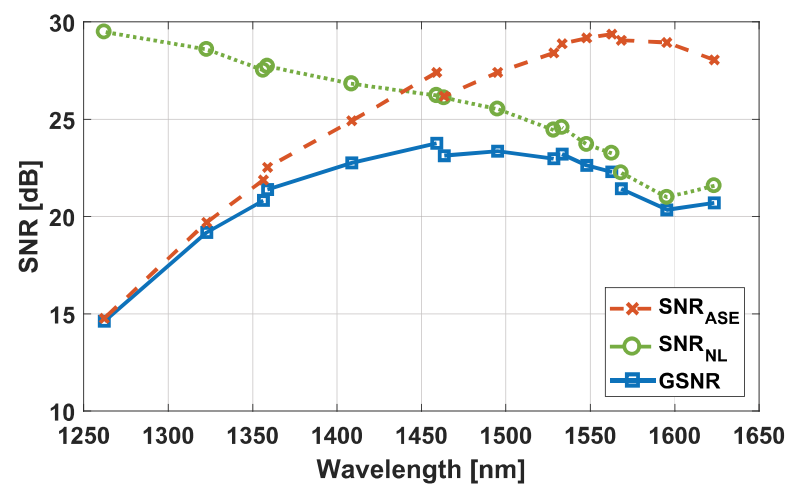

Fig. 8. Single span GSNR, $\mathrm{SNR}_{\mathrm{ASE}}$ and $\mathrm{SNR}_{\mathrm{NL}}$ in case all bands are used for channel transmission with $L_{s}=75 \mathrm{~km}$.

2) Transmission Scenarios \& Raman Amplifiers: The MBT system is composed of 5 bands. The full MBT is first explored (see Fig. 5(a)) and then the O-band off scenario is investigated and exploited for amplification of the E-band with Raman amplifiers. In this case, five Raman pumps are set over the entire O-band as displayed in Fig. 5(b). The pump configurations are reported in Table IV.

\section{NUMERICAL RESULTS}

Fig. 7 shows the signal power at the beginning of the fiber span, i.e., for $z=0$ (blue lines), and at the end, i.e. for $z=L_{S}$ (red lines), when all bands are used for data transmission. As expected the low-wavelength signals experience larger attenuation as the fiber loss is larger and the SRS depletes the channel power, on the contrary, high-wavelength channels present less attenuation as the attenuation is lower and the signals receive power from the lower wavelengths through the SRS. The fiber attenuation and the SRS induce an overall tilt of $8 \mathrm{~dB}$ after $50 \mathrm{~km}$ and of $10 \mathrm{~dB}$ after $75 \mathrm{~km}$ over the entire bandwidth. Fig. 8 shows the GSNR (solid line), the SNR $_{\text {ASE }}$ (dashed line) and the $\mathrm{SNR}_{\mathrm{NL}}$ (dash-dotted line) after a $75 \mathrm{~km}$ span when all bands are exploited for data transmission. Most transmission bands present strong unbalancing between ASE noise and NLI generation, despite the application of the LOGO strategy. This is because of the power transfer caused by the SRS towards the lower frequencies. Thus, an ASE limited transmission regime is observed at the high-frequencies, especially in the O-band, while the S-, C- and L-bands are mainly limited by the non-linear Kerr effect. The crossing point between $\mathrm{SNR}_{\mathrm{ASE}}$ and $\mathrm{SNR}_{\mathrm{NL}}$ is $\sim 1450 \mathrm{~nm}$, i.e., between E- and S-bands. For this reason, techniques such as the pre-emphasis of transmitted power levels, optimized Raman pump power and wavelength selection could be explored to mitigate the impact of SRS [59], [60]. The L-band has a slight upturn at the end of the band. This is due to the absence of channels on one side of the band which decreases the NLI generated in that portion of the band. The $\mathrm{SNR}_{\mathrm{ASE}}$ presents some discontinuities among the transmission bands one is clearly observable at $1450 \mathrm{~nm}$. 


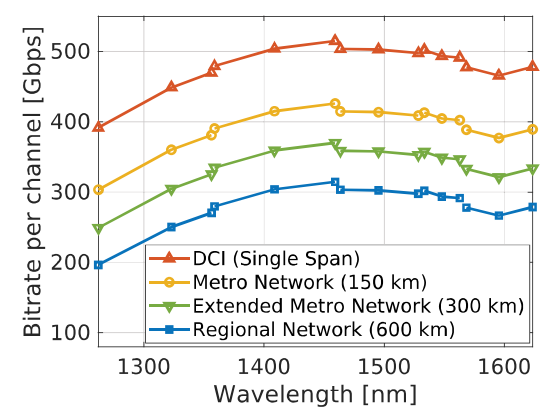

(a) $\mathrm{O} \rightarrow \mathrm{L}, L_{s}=50 \mathrm{~km}$

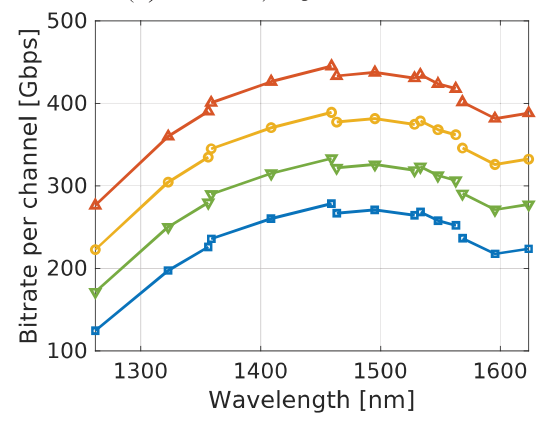

(d) $\mathrm{O} \rightarrow \mathrm{L}, L_{s}=75 \mathrm{~km}$

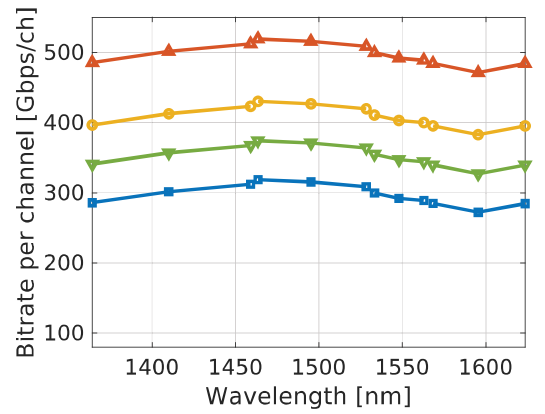

(b) No Raman, E $\rightarrow \mathrm{L}, L_{s}=50 \mathrm{~km}$

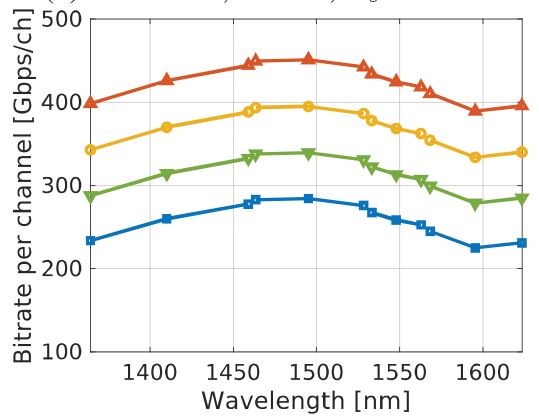

(e) No Raman, E $\rightarrow \mathrm{L}, L_{s}=75 \mathrm{~km}$
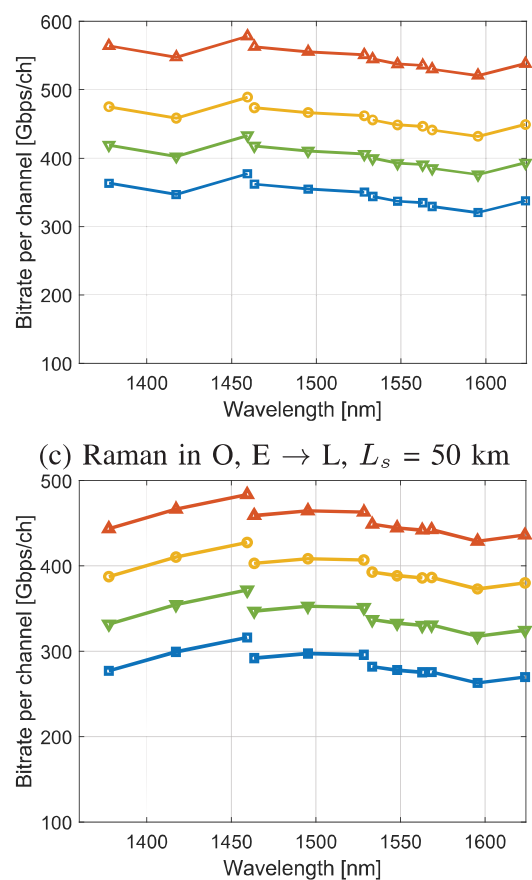

(f) Raman in $\mathrm{O}, \mathrm{E} \rightarrow \mathrm{L}, L_{s}=75 \mathrm{~km}$

Fig. 9. Net bitrate per wavelength for each scenario when considering two different span lengths. Each row reports different span lengths $-L_{s}=50 \mathrm{~km}$ the first and $L_{s}=75 \mathrm{~km}$ the second one. Each column refers to different bandwidth uses: full $\mathrm{O} \rightarrow \mathrm{L}$ the first, $\mathrm{E} \rightarrow \mathrm{L}$ without Raman amplification the second and $\mathrm{E} \rightarrow \mathrm{L}$ with Raman amplification the last one.

TABLE VI

GAIN FACTOR With RESPECT TO FULl C-BAND AND FULl C+L

\begin{tabular}{|c|c|c|c|c|c|c|c|}
\hline & & \multicolumn{3}{|c|}{ Gain from C-band } & \multicolumn{3}{|c|}{ Gain from C+L-band } \\
\hline & & $\mathrm{O} \rightarrow \mathrm{L}$ & $\begin{array}{c}\text { No Raman } \\
\mathrm{E} \rightarrow \mathrm{L}\end{array}$ & $\begin{array}{c}\text { Raman in } \mathrm{O} \\
\mathrm{E} \rightarrow \mathrm{L}\end{array}$ & $\mathrm{O} \rightarrow \mathrm{L}$ & $\begin{array}{c}\text { No Raman } \\
\mathrm{E} \rightarrow \mathrm{L}\end{array}$ & $\begin{array}{c}\text { Raman in } \mathrm{O} \\
\mathrm{E} \rightarrow \mathrm{L}\end{array}$ \\
\hline \multirow{4}{*}{$L_{s}=50 \mathrm{~km}$} & DCI & $10 \times$ & $8 \times$ & $8 \times$ & $4 \times$ & $3 \times$ & $3 \times$ \\
\hline & Metro & $10 \times$ & $7 \times$ & $8 \times$ & $4 \times$ & $3 \times$ & $7 \times$ \\
\hline & Extended Metro & $9.5 \times$ & $7 \times$ & $8 \times$ & $4 \times$ & $3 \times$ & $3 \times$ \\
\hline & Regional & $9 \times$ & $7 \times$ & $8 \times$ & $4 \times$ & $3 \times$ & $3 \times$ \\
\hline \multirow{4}{*}{$L_{s}=75 \mathrm{~km}$} & DCI & $9 \times$ & $7 \times$ & $7 \times$ & $4 \times$ & $3 \times$ & $3 \times$ \\
\hline & Metro & $9 \times$ & $7 \times$ & $7 \times$ & $3 \times$ & $2.7 \times$ & $3 \times$ \\
\hline & Extended Metro & $9 \times$ & $7 \times$ & $7 \times$ & $3 \times$ & $3 \times$ & $3 \times$ \\
\hline & Regional & $8 \times$ & $7 \times$ & $7 \times$ & $3 \times$ & $3 \times$ & $3 \times$ \\
\hline
\end{tabular}

TABLE VII

MAXIMUM CAPACITY $[\mathrm{Tb} / \mathrm{s}]$

\begin{tabular}{c|c|c|c|c}
\hline & & $\mathrm{O} \rightarrow \mathrm{L}$ & $\begin{array}{c}\text { No Raman } \\
\mathrm{E} \rightarrow \mathrm{L}\end{array}$ & $\begin{array}{c}\text { Raman in O } \\
\mathrm{E} \rightarrow \mathrm{L}\end{array}$ \\
\hline \multirow{3}{*}{$L_{s}=50 \mathrm{~km}$} & DCI & 450 & 348 & 359 \\
& Metro & 367 & 268 & 302 \\
& Extended Metro & 314 & 229 & 265 \\
& Regional & 263 & 208 & 229 \\
\hline \multirow{3}{*}{$L_{s}=75 \mathrm{~km}$} & DCI & 375 & 296 & 298 \\
& Metro & 323 & 258 & 261 \\
& Extended Metro & 272 & 219 & 225 \\
& Regional & 222 & 181 & 189 \\
\hline
\end{tabular}

Table $\mathrm{V}$ and Fig. 9 report the average spectral efficiency per band and bitrate per channel, respectively, attainable in each of the transmission bands when considering four different transmission scenarios with span lengths of $50 \mathrm{~km}$ and $75 \mathrm{~km}$ : single span DCI, $150 \mathrm{~km}$ metro, $300 \mathrm{~km}$ extended metro and $600 \mathrm{~km}$ regional optical networks. Three different transmission configurations are considered for each case: data transmission over the full MBT spectrum ("O $\rightarrow$ L"); data transmission from the E- to L-band only and without Raman amplification ("No Raman, E $\rightarrow$ L"); and finally data transmission using the E- to L-bands with Raman pumps placed in the O-band ("Raman in $\mathrm{O}, \mathrm{E} \rightarrow \mathrm{L}$ "). Further details on the computation of the average spectral efficiency per band are shown in Appendix C.

Results reported in Fig. 9 show that the best performing channels are the ones in the middle of the MBT WDM comb, which correspond to the best balancing between $\mathrm{SNR}_{\mathrm{ASE}}$ and $\mathrm{SNR}_{\mathrm{NL}}$, as reported in Fig. 8. Extending the fiber span leads to a decrease of the maximum throughput per channel, as expected, due to the higher accumulated loss which decreases the $\mathrm{SNR}_{\mathrm{ASE}}$. Meanwhile, the NLI and SRS cross-talk do not 
$\square \rightarrow \mathrm{L} \quad$ No Raman, $\mathrm{E} \rightarrow \mathrm{L} \quad$ Raman in $\mathrm{O}, \mathrm{E} \rightarrow \mathrm{L}$

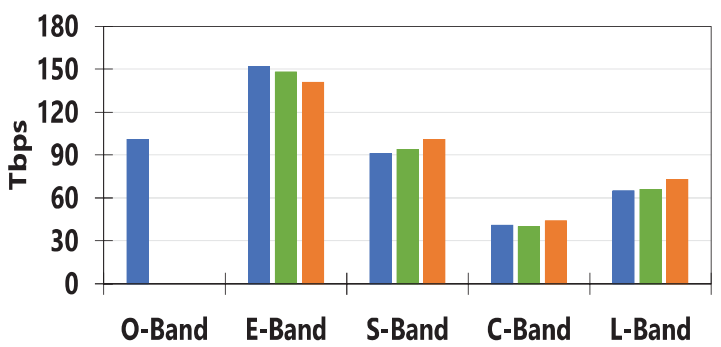

(a) DCI (Single Span), $L_{s}=50 \mathrm{~km}$

$\square \mathrm{O} \rightarrow \mathrm{L} \quad$ No Raman, $\mathrm{E} \rightarrow \mathrm{L} \quad$ Raman in $\mathrm{O}, \mathrm{E} \rightarrow \mathrm{L}$

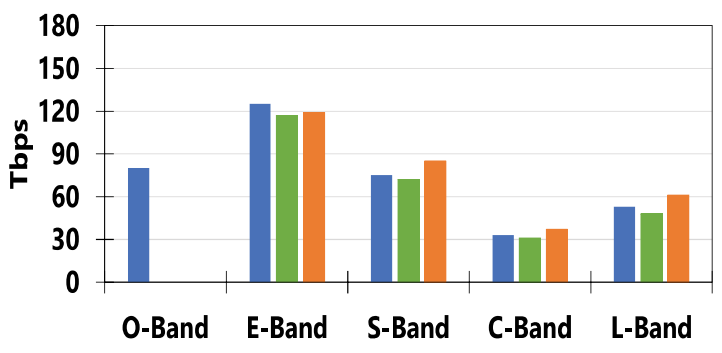

(c) Metro $(150 \mathrm{~km}), L_{s}=50 \mathrm{~km}$

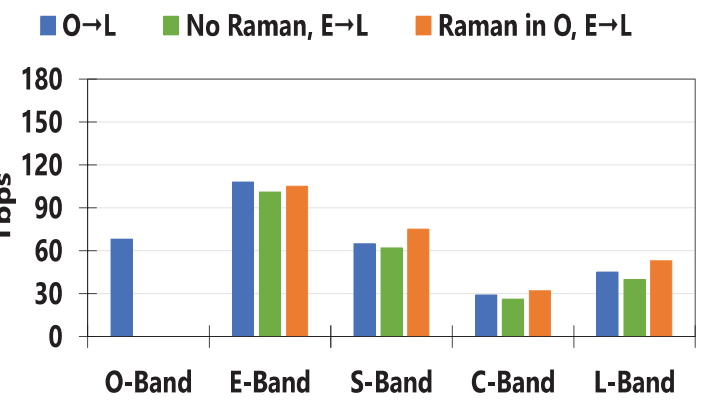

(e) Extended Metro (300 km), $L_{s}=50 \mathrm{~km}$

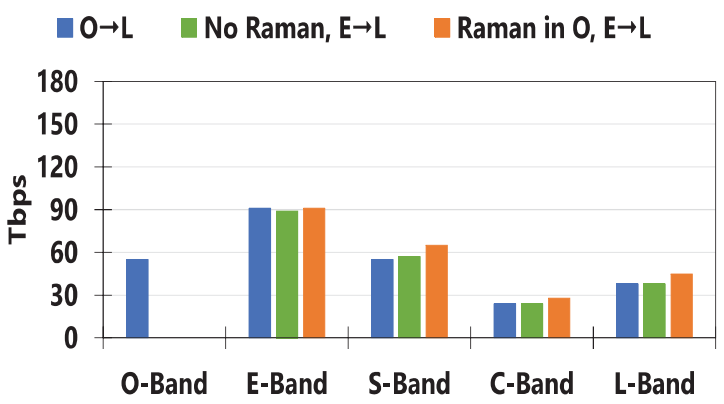

(g) Regional $(600 \mathrm{~km}), L_{s}=50 \mathrm{~km}$ $\square$ - No Raman, $\mathrm{E} \rightarrow \mathrm{L} \quad$ Raman in $\mathrm{O}, \mathrm{E} \rightarrow \mathrm{L}$

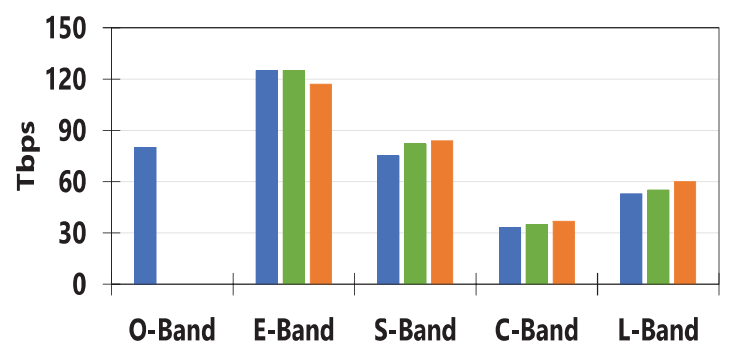

(b) DCI (Single Span), $L_{s}=75 \mathrm{~km}$

$\square \mathrm{O} \rightarrow \mathrm{L} \quad$ No Raman, $\mathrm{E} \rightarrow \mathrm{L} \quad$ Raman in $\mathrm{O}, \mathrm{E} \rightarrow \mathrm{L}$

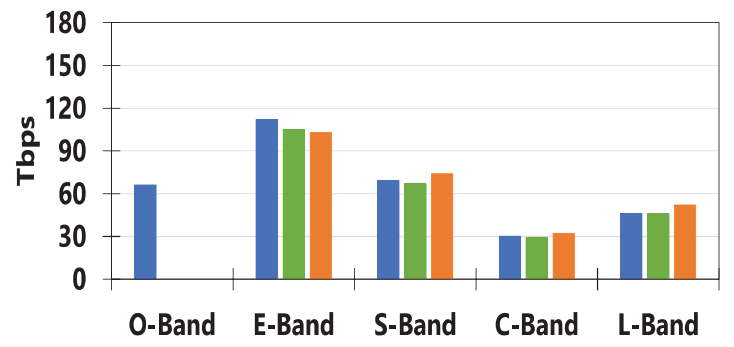

(d) Metro $(150 \mathrm{~km}), L_{s}=75 \mathrm{~km}$

\section{$\square \mathrm{O} \rightarrow \mathrm{L} \quad$ No Raman, $\mathrm{E} \rightarrow \mathrm{L} \quad$ Raman in $\mathrm{O}, \mathrm{E} \rightarrow \mathrm{L}$}

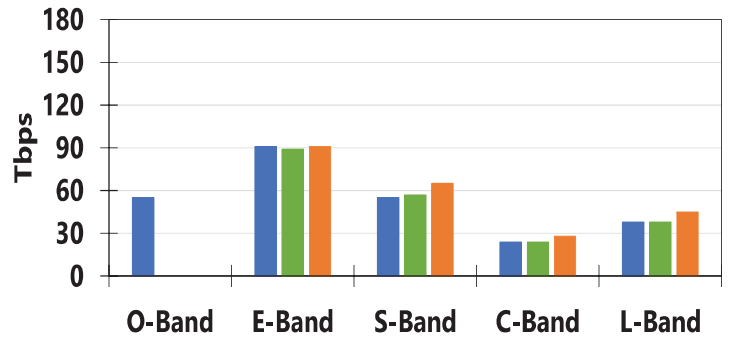

(f) Extended Metro (300 km), $L_{s}=75 \mathrm{~km}$

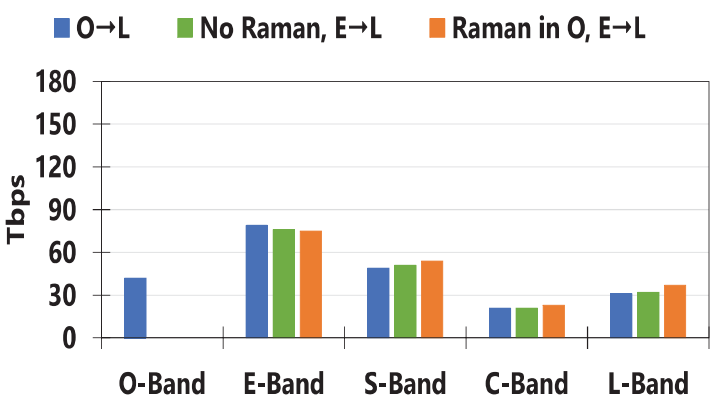

(h) Regional $(600 \mathrm{~km}), L_{s}=75 \mathrm{~km}$

Fig. 10. Maximum capacity per transmission band. Each row refers to a different reach: DCI (75 km) the first, metro (150 km) the second, extended metro $(300 \mathrm{~km})$ the third and regional $(600 \mathrm{~km})$ the last one. Left-hand side: $50 \mathrm{~km}$ spans; and right-hand side: $75 \mathrm{~km}$ spans.

considerably vary with the fiber length, provided that the effective length is exceeded, as in the analyzed scenario. So, the spectral dependence of the maximum throughput remains practically unchanged. The discontinuities among transmission bands result from the different amplification technologies assumed for each band. These are summarized by different noise figures, as reported in Table II. As previously stated, different power levels are set for each band according to the LOGO strategy, so depending on fiber parameters - length, $\alpha(\lambda), D(\lambda)$ and $\gamma-$ and on the amplifier parameters gain and noise figure [46]. The O-band shows the worst performance, when compared to the remaining transmission bands as a consequence of the higher 
fiber attenuation, higher noise figure of the amplifiers, and high power depletion induced by inter-channel SRS. Thus, whether to exploit the O-band in MBT systems has to be analyzed from a techno-economic perspective for specific use cases. Another option for the use of the O-band is to allocate Raman pumps to improving performance in the remaining highest-frequency band (see Fig. 5) - the E-band - that is largely depleted by the SRS power transfer and consequently dominated by ASE noise.

Table VII and Fig. 10 report the maximum capacity per band and maximum total capacity, respectively, for each transmission scenario. These results show that Raman amplification enables a limited throughput increase, mainly over longer fiber spans, because of the large loss in the O-band that limits the Raman efficiency. So, as for C-band only transmission systems, Raman amplification is beneficial only for very-long fiber spans. An improvement of the maximum throughput due to Raman amplification limited to $10 \%$, was observed in all cases.

Fig. 10 shows that $\mathrm{O}$ - and S-band carry a comparable total traffic, despite the higher number of wavelengths in O-band: 240 versus 182. This result is a consequence of the lower GSNR in the O-band, which provides a smaller bitrate per wavelength. Despite the much worse optical performance, the O-band can transport a higher total throughput with respect to C-band, only due to the much wider bandwidth ( $\sim 4$ times wider), so ts actual usability must follow an accurate techno-economics analysis. Table VII shows that by increasing the span length from $50 \mathrm{~km}$ to $75 \mathrm{~km}$ in the DCI scenario, the maximum throughput decreases by $75 \mathrm{~Tb} / \mathrm{s}$ from $450 \mathrm{~Tb} / \mathrm{s}$ to $375 \mathrm{~Tb} / \mathrm{s}$, when the full MBT from $\mathrm{O}$ - to L-band is assumed. This value corresponds to a decrease of the SMF throughput of about $15 \%$, highlighting the high impact of the DCI link length on performance. A smaller impact of the span length on the maximum throughput is observed for the remaining transmission scenarios, as expected, since the optical performance / maximum throughput is already limited for the reference scenario: span length of $50 \mathrm{~km}$. Additionally, Table VII displays that using O-band for data transport can be indeed interesting for the DCI transmission case with $50 \mathrm{~km}$ span length, as it enables almost $30 \%$ of additional throughput. Even for the longer fiber length of $75 \mathrm{~km}$, when the O-band is used for Raman pumping instead of data transport, the overall throughput is reduced roughly by $20 \%$. Therefore, the use of the O-band for data transport enables a large additional throughput in DCI scenarios, but with limited spectral efficiency, at the cost of a very large number of transceivers. So, the use of the O-band for intense data transport seems to be motivated only in case it is not possible to install new cables and low-cost transponders are available. Further techno-economic analysis is clearly required, in particular to investigate if there exist solid use cases to open up the O-band for transmission. Finally, Table VI reports the throughput increase with respect to the use of full C-band and full $\mathrm{C}+\mathrm{L}-\mathrm{b}$ and under the same conditions. As expected, the throughput gain is always smaller than the bandwidth enlargements as the GSNR decreases enlarging the bandwidth. Furthermore, the gain always decreases when propagation distances become larger and when the span length is larger. In general a full $\mathrm{O} \rightarrow \mathrm{L}$ MBT can carry a traffic 8 to 10 times larger than a full C-band transmission and 2.5 to 3 times more than a full $\mathrm{C}+\mathrm{L}-$ band MBT.

\section{CONCLUSION AND OUTLOOK}

The currently exploited C-band is a minor portion of the available single-mode low-loss spectrum available for transmission over ITU-T G.652 single-mode fibers. The vast majority of optical fibers deployed in the installed network infrastructures do not present anymore the $\mathrm{OH}$ absorption peak in the E-band - as reported in the cited references - and so are ITU-T G.652.D fibers. Consequently, the entire single mode spectrum, from the $\mathrm{O}$ - to the L-band, could be exploited for transmission once the technology become available relying on a fiber loss always smaller than $0.4 \mathrm{~dB} / \mathrm{km}$. High-bandwidth demand and short-distance transmission scenarios, such as DCI with distances $\leq 40 \mathrm{~km}$, in case of absence of available dark fibers, might represent the starting use cases where MBT could be first deployed. We carried out an extensive analysis, by estimating the generalized SNR, to evaluate the multi-band feasible throughput of the most widely deployed fiber, the ITU-T G.652.D. We aimed at estimating the throughput limits set by fiber propagation to provide a reference assessment for MBT techno-economics including different components' options, so we deliberately made simplifying hypotheses on the components to focus on the fiber transmission limitations. We considered four relevant scenarios, with transmission distances ranging from $50 \mathrm{~km}$ up to $600 \mathrm{~km}$, and two different span lengths, $50 \mathrm{~km}$ and $75 \mathrm{~km}$. Given these assumptions, our investigations show that multi-band transmission may be a feasible alternative to widely researched approaches based on multi-mode and/or multi-core fibers, because it does not require the deployment of new cables. Therefore, we can conclude that the most efficient option, once dark-fibers and space within ducts is exhausted, is to install multi-band transmission covering the entire low-loss spectrum of SMF. This process is starting with the enabling of the already commercial available L-band, and we envision it will possibly continue by populating the entire low-loss spectrum of SMF, in case it is needed. To afford the ever growing traffic demand, MBT will not be sufficient and multiple fiber transmission will be a firm request. But, the use of MBT in cables including multiple fibers will enlarge the throughput per fiber, consequently enlarging the transmission spatial efficiency in bit $/ \mathrm{s} / \mathrm{m}^{2}$, so enabling a better exploitation of the precious space available in fiber ducts that requires large CAPEX investments.

MBT has the potential to guarantee a throughput per fiber above $450 \mathrm{~Tb} / \mathrm{s} /$ fiber over DCI distances of $\sim 50 \mathrm{~km}$, which is approximately a $10 \times$ increase compared to the best commercial C-band systems [1]. In case of regional / long-haul distances, MBT may achieve a throughput well above $200 \mathrm{~Tb} / \mathrm{s} /$ fiber. In general, a full $\mathrm{O} \rightarrow \mathrm{L} \mathrm{MBT}$ can accommodate $\sim 8 / 10 \times$ more traffic than the only $\mathrm{C}$-band and $\sim 2.5 / 3 \times$ more than $\mathrm{C}+\mathrm{L}$-band. Further improvements such as interleaving signals and Raman pumps can be exploited to further enhance the propagation performance as shown in [59], [60].

\section{APPENDIX A}

The effect of SRS is modelled according to [61] and the Rayleigh back-scattering is not taken into account as it has been demonstrated that it is negligible in Silica-Core Fibers (SCF) with pumps having power lower than $800 \mathrm{~mW}$ [62]. The power 
evolution $P\left(z, f_{i}\right)$ of each signal and Raman pump is described by the following system of $N$ ordinary differential equations (ODE):

$$
\left\{\begin{aligned}
\pm \frac{d P\left(z, f_{1}\right)}{d z}= & -\alpha\left(f_{1}\right) P\left(z, f_{1}\right) \\
& +\sum_{j=2}^{N} C_{R}\left(f_{1}, f_{n}\right) P\left(z, f_{j}\right) P\left(z, f_{1}\right) \\
\pm \frac{d P\left(z, f_{i}\right)}{d z}= & -\alpha\left(f_{i}\right) P\left(z, f_{i}\right) \\
& +\sum_{j=i+1}^{N} C_{R}\left(f_{i}, f_{n}\right) P\left(z, f_{j}\right) P\left(z, f_{i}\right) \\
& -\sum_{j=1}^{i-1} \frac{f_{i}}{f_{j}} C_{R}\left(f_{i}, f_{n}\right) P\left(z, f_{j}\right) P\left(z, f_{i}\right) \\
& \cdots \\
\pm \frac{d P\left(z, f_{N}\right)}{d z}= & -\alpha\left(f_{i}\right) P\left(z, f_{N}\right) \\
& -\sum_{j=1}^{N-1} \frac{f_{N}}{f_{j}} C_{R}\left(f_{i}, f_{n}\right) P\left(z, f_{j}\right) P\left(z, f_{N}\right)
\end{aligned}\right.
$$

Each equation describes the power evolution along the spatial variable $z$ of the signal or the pump at the $i$-th frequency $f_{i}$, with $f_{i}<f_{j}$ for $i<j$. Thus, $f_{1}$ is the lower frequency, while $f_{N}$ is the larger. $N$ is the number of signal and Raman pumps. The \pm sign in each equation indicates co-propagation if " + " or counter-propagation if "-" of the $i$-th signal or Raman pump. $\alpha\left(f_{i}\right)$ is the fiber attenuation coefficient at the frequency $f_{i}$, $C_{R}\left(f_{i}, f_{n}\right)$ is the Raman gain efficiency between the frequencies $f_{i}$ and $f_{n}$. The boundary conditions of the problem are given by the transmitted power $P\left(z_{0}, f_{i}\right)$ of each signal and Raman pump at the transmission point $z_{0}$. $z_{0}$ is equal to 0 for all co-propagating Raman pumps and for all signals (being co-propagating by definition), while $z_{0}$ is equal to the span length $L_{S}$ for all counter-propagating Raman pumps. Thus, the problem is described by a system of coupled ordinary differential equations (ODE) with two points boundary conditions $\left(z_{0}=0\right.$ and $\left.z_{0}=L_{S}\right)$. Since, in general, there is not an analytic solution, a numerical ODE solver is used to compute $P\left(z, f_{i}\right)$. Finally, the overall frequency and space dependent fiber loss profile $\rho(z, f)$ is computed as:

$$
\rho\left(z, f_{i}\right)=\sqrt{\frac{P\left(z, f_{i}\right)}{P\left(z_{0}, f_{i}\right)}}
$$

\section{APPENDIX B}

Each span of the transmission line is modeled according to the equivalent block diagram reported in Fig. 6. The WDM signal in each band is multiplexed using a multiband multiplexer and the resulting signal is propagated through the fiber which is characterized by a transfer function $\rho\left(L_{S}, f\right)$. This transfer function includes both the fiber attenuation and the SRS. The impact of NLI is added to the signal at fiber output. Afterwards, the channels are demultiplexed using a MBT demultiplexer. Each band is then amplified via lumped amplification and a GFF is applied to the signal. Finally, ASE noise is added. For each WDM channel, the GSNR is computed as:

$$
\mathrm{GSNR}=\frac{P_{S}}{P_{\mathrm{ASE}}+P_{\mathrm{NLI}}}
$$

where, $P_{S}$ is the signal power, $P_{\mathrm{ASE}}$ is the ASE noise, defined as

$$
P_{\mathrm{ASE}}=h G_{e q} F_{e q} f B_{\mathrm{ref}}
$$

where $h$ is the Planck constant, $f$ is the frequency, $B_{\text {ref }}$ is the reference noise bandwidth. The reference bandwidth is equal to the signal symbol rate $R_{S}$. $G_{e q}$ and $F_{e q}$ are the equivalent Raman-EDFA gain and noise figure, respectively. In case Raman amplification is not used, $G_{e q}$ and $F_{e q}$ correspond to the gain and the noise figure of lumped amplifiers, respectively. Finally, $P_{\mathrm{NLI}}$ is

$$
P_{\mathrm{NLI}}=B_{\mathrm{ref}} G_{\mathrm{NLI}}\left(L_{s}, f\right),
$$

where $L_{s}$ is the span length and $G_{\mathrm{NLI}}\left(L_{s}, f\right)$ is the NLI power spectral density defined as reported in equation (1).

\section{APPENDIX C}

The average spectral efficiency per band is the average performance of the occupied band $B$ and it has been computed as

$$
\frac{\sum_{i=1}^{N_{c} h} R b_{n e t, i}}{B}=\frac{\sum_{n} \mathrm{SE}_{n} R_{S}}{N_{c h} \Delta f}
$$

where, $R b_{n e t, i}$ is the bitrate of the $n$-th channel, $R_{S}$ is the symbol rate, $N_{c h}$ is the number of channels, $\Delta f$ is the WDM grid size and $\mathrm{SE}$ is the channel net spectral efficiency computed as

$$
\mathrm{SE}=\frac{2 \log _{2}(1+\mathrm{SNR})}{1+O H},
$$

where $\mathrm{OH}$ is the overhead.

\section{REFERENCES}

[1] "Infinera Groove G30," 2019. [Online]. Available: https://www.infinera. $\mathrm{com} /$ infinera-sets-highest-performance-600g-transmission-record/

[2] "Cisco Visual Networking Index: Forecast and Methodology, June 2017," 2017. [Online]. Available: https://www.cisco.com/c/en/us/solutions/ service-provider/visual-networking-index-vni/index.html

[3] G. Wellbrock and T. J. Xia, "How will optical transport deal with future network traffic growth?" in Proc. IEEE Eur. Conf. Opt. Commun., Sep. 2014, pp. 1-3.

[4] P. J. Winzer, "Scaling optical networking technologies for next generation SDM," in Proc. Opt. Fiber Commun. Conf. Expo., 2018, Paper. Th1F.4.

[5] T. Jimenez, V. López, F. Jimenez, O. Gonzalez, and J. Fernandez, “Technoeconomic analysis of transmission technologies in low aggregation rings of metropolitan networks," in Proc. IEEE Opt. Fiber Commun. Conf. Exhib., 2017 , pp. 1-3.

[6] R. Van Uden et al., "Ultra-high-density spatial division multiplexing with a few-mode multicore fibre," Nature Photon., vol. 8, no. 11, pp. 865-870, 2014

[7] T. Kobayashi et al., "1-Pb/s (32 SDM/46 WDM/768 Gb/s) C-band dense SDM transmission over $205.6-\mathrm{km}$ of single-mode heterogeneous multicore fiber using 96-Gbaud PDM-16QAM channels," in Proc. Opt. Fiber Commun. Conf., 2017, Paper Th5B-1.

[8] A. Napoli et al., "Towards multiband optical systems," in Proc. Photon. Netw. Devices, 2018, Paper NeTu3E-1.

[9] "Handbook - Optical fibres, cables and systems - ITU, 2009," 2009. [Online]. Available: https://www.itu.int/dms_pub/itu-t/opb/hdb/T-HDBOUT.10-2009-1-PDF-E.pdf

[10] T. Miya, Y. Terunuma, T. Hosaka, and T. Miyashita, "Ultimate low-loss single-mode fibre at $1.55 \mu \mathrm{m}$, , Electron. Lett., vol. 15, no. 4, pp. 106-108, 1979

[11] "Prysmian, Broadband boost for Rural New Zealand," 2015. [Online]. Available: https://www.prysmiangroup.com/staticres/Nexst-20152/broadband-boost-for-rural-new-zealand.html

[12] J.-X. Cai et al., "70.4 Tb/s capacity over 7,600 km in C+ L band using coded modulation with hybrid constellation shaping and nonlinearity compensation," in Proc. Opt. Fiber Commun. Conf., 2017, Paper Th5B-2.

[13] J. Renaudier, "100 nm ultra-wideband optical fiber transmission systems using semiconductor optical amplifiers," in Proc. IEEE Eur. Conf. Opt. Commun., 2018, pp. 1-3. 
[14] "The Importance of International Standards in the Evolution of Telecommunications Networks, white paper," 2004. [Online]. Available: https://www.corning.com/content/dam/corning/media/worldwide/coc/ documents/Fiber/RC-\%20White\%20Papers/WP-General/WP5160.pdf

[15] "Increasing data traffic requires full spectral window usage in optical single-mode fiber cables, white paper," 2015. [Online]. Available: http://telecoms.com/wp-content/blogs.dir/1/files/2015/02/Whitepaper_Increasing-Data-Traffic_Final.pdf

[16] "CRU worldwide telecom cables market report," 2018. [Online]. Available: https://www.crugroup.com/analysi

[17] S. Okamoto, K. Horikoshi, F. Hamaoka, K. Minoguchi, and A. Hirano, "5-band (O, E, S, C, and L) WDM transmission with wavelength adaptive modulation format allocation," in Proc. 42nd Eur. Conf. Opt. Commun., 2016, pp. 1-3.

[18] G.-H. Gho, L. Klak, and J. M. Kahn, "Rate-adaptive coding for optical fiber transmission systems," J. Lightw. Technol., vol. 29, no. 2, pp. 222-233, Jan. 2011.

[19] A. Pilipetskii et al., "The subsea fiber as a shannon channel," in Proc. Suboptic, 2019, pp. 1-7.

[20] M. Filer, M. Cantono, A. Ferrari, G. Grammel, G. Galimberti, and V. Curri, "Multi-vendor experimental validation of an open source qot estimator for optical networks," J. Lightw. Technol., vol. 36, no. 15, pp. 3073-3082, Aug. 2018

[21] A. Ferrari et al., "GNPY: An open source application for physical layer aware open optical networks," J. Opt. Commun. Netw., vol. 12, no. 6, pp. C31-C40, 2020.

[22] A. Ferrari et al., "Experimental validation of an open source quality of transmission estimator for open optical networks," in Proc. Opt. Fiber Commun. Conf., 2020, Paper W3C.2. [Online]. Available: http://www. osapublishing.org/abstract.cfm?URI=OFC-2020-W3C.2

[23] M. Cantono, J. L. Auge, and V. Curri, "Modelling the impact of SRS on NLI generation in commercial equipment: An experimental investigation," in Proc. Opt. Fiber Commun. Conf., 2018, Paper M1D-2.

[24] M. Cantono, D. Pilori, A. Ferrari, and V. Curri, "Introducing the Generalized GN-model for Nonlinear Interference Generation including space/frequency variations of loss/gain," 2017, arXiv:1710.02225.

[25] M. Cantono et al., "On the interplay of nonlinear interference generation with stimulated raman scattering for qot estimation," J. Lightw. Technol., vol. 36, no. 15, pp. 3131-3141, Aug. 2018.

[26] I. Roberts, J. M. Kahn, J. Harley, and D. W. Boertjes, "Channel power optimization of WDM systems following Gaussian noise nonlinearity model in presence of stimulated raman scattering," J. Lightw. Technol., vol. 35, no. 23, pp. 5237-5249, Dec. 2017.

[27] D. Semrau, R. I. Killey, and P. Bayvel, "The gaussian noise model in the presence of inter-channel stimulated raman scattering," J. Lightw. Technol., vol. 36, no. 14, pp. 3046-3055, Jul. 2018.

[28] J. K. Fischer et al., "Maximizing the capacity of installed optical fiber infrastructure via wideband transmission," in Proc. IEEE 20th Int. Conf. Transparent Opt. Netw., 2018, pp. 1-4.

[29] A. Ferrari et al., "Upgrade capacity scenarios enabled by multi-band optical systems," in Proc. ICTON, 2019, pp. 1-4.

[30] P. Poggiolini et al., "The logon strategy for low-complexity control plane implementation in new-generation flexible networks," in Proc. IEEE Opt. Fiber Commun. Conf., 2013, pp. 1-3.

[31] A. Napoli et al., "Perspectives of multi-band optical communication systems," in Proc. OECC, 2018, pp. 1-2.

[32] Fiberlabs-Inc, "Praseodymium fluoride fiber glass doped amplifier," 2018. [Online]. Available: www.fiberlabs.com

[33] E. Dianov, "Bismuth-doped optical fibers: A challenging active medium for near-IR lasers and optical amplifiers," Light: Sci. Appl., vol. 1, pp. e12-e12, 2012

[34] S. Aozasa et al., "TM-doped fiber amplifiers for 1470-nm-band WDM signals," PTL, vol. 12, no. 10, pp. 1331-1333, 2000

[35] V. Curri and A. Carena, "Merit of raman pumping in uniform and uncompensated links supporting NyWDM transmission," J. Lightw. Technol., vol. 34, no. 2, pp. 554-565, Jan. 2016.

[36] L. Krzczanowicz et al., "Low transmission penalty dual-stage broadband discrete raman amplifier," Opt. Express, vol. 26, no. 6, pp. 7091-7097, 2018.

[37] "MPB-Communications, technical data sheets," 2018. [Online]. Available: https://www.globenewswire.com/news-release/2018/10/03/1600666/0/ en/Windstream-Deploys-Infinera-C-L-Solution-Sets-Foundation-toDouble-Fiber-Capacity.html

[38] F. Hamaoka et al., "150.3-Tb/s ultra-wideband (S, C, and L bands) singlemode fibre transmission over 40-km using $>519 \mathrm{~GB} / \mathrm{s} / \mathrm{a}$ PDM-128QAM signals," in Proc. IEEE Eur. Conf. Opt. Commun., 2018, pp. 1-3.
[39] F. Hamaoka et al., "Ultra-wideband WDM transmission in S, C, and L-bands using signal power optimization scheme," J. Lightw. Technol., vol. 37, no. 8, pp. 1764-1771, Apr. 2019.

[40] C. Doerr et al., "O, E, S, C, and L band silicon photonics coherent modulator/receiver," in Proc. IEEE Opt. Fiber Commun. Conf. Exhib., 2016, pp. 1-3.

[41] M. Ionescu et al., " $91 \mathrm{~nm} \mathrm{C+L} \mathrm{hybrid} \mathrm{distributed} \mathrm{raman/erbium-doped}$ fibre amplifier for high capacity subsea transmission,", in Proc. Eur. Conf. Opt. Commun., Sep. 2018, pp. 1-3.

[42] M. Filer, J. Gaudette, Y. Yin, D. Billor, Z. Bakhtiari, and J. L. Cox, "Lowmargin optical networking at cloud scale," IEEE/OSA J. Opt. Commun. Netw., vol. 11, no. 10, pp. C94-C108, Oct. 2019.

[43] R. Schmogrow et al., "Error vector magnitude as a performance measure for advanced modulation formats," IEEE Photon. Technol. Lett., vol. 24, no. 1, pp. 61-63, Jan. 2012.

[44] A. Carena, V. Curri, G. Bosco, P. Poggiolini, and F. Forghieri, "Modeling of the impact of nonlinear propagation effects in uncompensated optical coherent transmission links," J. Lightw. Technol., vol. 30, no. 10, pp. 1524-1539, May 2012

[45] A. Splett, C. Kurtzke, and K. Petermann, "Ultimate transmission capacity of amplified optical fiber communication systems taking into account fiber nonlinearities," in Proc. IEEE 19th Eur. Conf. Opt. Commun., 1993, pp. 4144.

[46] V. Curri et al., "Design strategies and merit of system parameters for uniform uncompensated links supporting nyquist-WDM transmission," $J$. Lightw. Technol., vol. 33, no. 18, pp. 3921-3932, Sep. 2015.

[47] K. Minoguchi et al., "Experiments on stimulated raman scattering in s-and 1-bands 16-QAM signals for ultra-wideband coherent WDM systems," in Proc. Opt. Fiber Commun. Conf., 2018, Paper Th1C-4.

[48] G. Saavedra et al., "Inter-channel stimulated raman scattering and its impact in wideband transmission systems," in Proc. IEEE Opt. Fiber Commun. Conf. Expo., 2018, pp. 1-3.

[49] V. Curri, A. Carena, P. Poggiolini, G. Bosco, and F. Forghieri, "Extension and validation of the gn model for non-linear interference to uncompensated links using raman amplification," Opt. Express, vol. 21, no. 3, pp. 3308-3317, 2013.

[50] V. Curri and A. Carena, "Merit of raman pumping in uniform and uncompensated links supporting nywdm transmission," J. Lightw. Technol., vol. 34, no. 2, pp. 554-565, Jan. 2016.

[51] S. Kado, Y. Emori, S. Namiki, N. Tsukiji, J. Yoshida, and T. Kimura, "Broadband flat-noise raman amplifier using low-noise bidirectionally pumping sources," in Proc. IEEE 27th Eur. Conf. Opt. Commun., 2001, vol. 6, pp. 38-39.

[52] M. Cantono, J. L. Auge, and V. Curri, "Modelling the impact of SRS on NLI generation in commercial equipment: An experimental investigation," in Proc. Opt. Fiber Commun. Conf., 2018, Paper M1D.2.

[53] M. Cantono et al., "On the interplay of nonlinear interference generation with stimulated raman scattering," J. Lightw. Technol., vol. 36, no. 15, pp. 3131-3141, Aug. 2018.

[54] D. Semrau, E. Sillekens, R. I. Killey, and P. Bayvel, "The ISRS GN model, an efficient tool in modeling ultra-wideband transmission in point-to-point and network scenarios," in Proc. Eur. Conf. Opt. Commun., Sep. 2018, pp. $1-3$.

[55] J.-C. Bouteiller, L. Leng, and C. Headley, "Pump-pump four-wave mixing in distributed raman amplified systems," J. Lightw. Technol., vol. 22, no. 3, pp. 723-732, Mar. 2004

[56] I. Sackey et al., "Characterization of polarization insensitive fopa using a polarization-maintaining highly non-linear fiber," in Proc. Opt. Fiber Commun. Conf., 2013, Paper OTu2D-2.

[57] "MPB-Communications, technical data sheets," 2020. [Online]. Available: http://mpbcommunications.com/en/site/products/network_ready_ telecom_solutions/2RU-Series/raman_pumps/index.html

[58] P. J. Winzer, D. T. Neilson, and A. R. Chraplyvy, "Fiber-optic transmission and networking: The previous 20 and the next 20 years," Opt. Express, vol. 26, no. 18, pp. 24 190-24 239, 2018

[59] T. Naito, T. Tanaka, K. Torii, N. Shimojoh, H. Nakamoto, and M. Suyama, "A broadband distributed raman amplifier for bandwidths beyond $100 \mathrm{~nm}$," in Proc. IEEE Opt. Fiber Commun. Conf. Exhibit, 2002, pp. 116-117.

[60] A. Gnauck, R. Jopson, and P. Winzer, "Demonstration of counterpropagating raman pump placed near signal-channel wavelengths," IEEE Photon. Technol. Lett., vol. 29, no. 1, pp. 154-157, Jan. 2017.

[61] J. Bromage, "Raman amplification for fiber communications systems," $J$. Lightw. Technol., vol. 22, no. 1, pp. 79-93, Jan. 2004.

[62] P. Hansen et al., "Rayleigh scattering limitations in distributed raman preamplifiers," IEEE Photon. Technol. Lett., vol. 10, no. 1, pp. 159-161, Jan. 1998. 